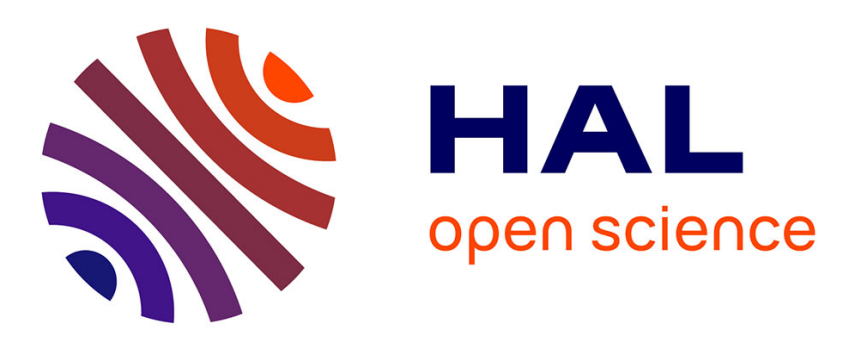

\title{
Une révision de la séquence de la Riera (Asturies) et la question du Badegoulien cantabrique
}

Bruno Bosselin, François Djindjian

\section{To cite this version:}

Bruno Bosselin, François Djindjian. Une révision de la séquence de la Riera (Asturies) et la question du Badegoulien cantabrique. Bulletin de la Société préhistorique française, 1999, 96 (2), pp.153-173. hal-00201603

\section{HAL Id: hal-00201603 \\ https://hal.science/hal-00201603}

Submitted on 15 Jan 2008

HAL is a multi-disciplinary open access archive for the deposit and dissemination of scientific research documents, whether they are published or not. The documents may come from teaching and research institutions in France or abroad, or from public or private research centers.
L'archive ouverte pluridisciplinaire HAL, est destinée au dépôt et à la diffusion de documents scientifiques de niveau recherche, publiés ou non, émanant des établissements d'enseignement et de recherche français ou étrangers, des laboratoires publics ou privés. 


\title{
Bruno BOSSELIN et François DJINDJIAN du Badegoulien cantabrique
}

\section{Une révision}

de la séquence de la Riera (Asturies) et la question

\begin{abstract}
Résumé
Une nouvelle interprétation de la séquence de la Riera (Asturies) est proposée à partir d'une analyse pluridisciplinaire incluant le paléoenvironnement, l'approvisionnement en matières premières lithiques, les témoins du débitage et les industries lithiques et osseuses. Après un bref rappel sur le modèle établi par L.G. Straus, la révision du cadre paléoclimatique souligne un contexte steppique pour l'ensemble de la stratigraphie, avec quelques épisodes plus humides, hormis le sommet de la séquence correspondant au réchauffement tardiglaciaire (“Bølling/Allerød"). L'analyse des matières premières et du débitage met en évidence une économie de subsistance essentiellement locale pour la fin du Solutréen et le Badegoulien, coïncidant avec le maximum glaciaire, au contraire du début du Solutréen et du Magdalénien où une circulation le long de la côte cantabrique et jusqu'au sud du bassin aquitain est envisageable. Les résultats du traitement statistique des industries lithiques révèlent la présence de plusieurs faciès industriels en succession stratigraphique, dont une entité nouvelle: le Badegoulien cantabrique. Une étude typologique démontre l'existence de continuités et de discontinuités entre ces différents faciès. L'industrie osseuse souligne par ailleurs de fortes convergences entre le Solutréen et le Badegoulien. Cette structuration est ensuite confrontée aux autres données situées entre 22000 et 16000 B.P. dans les Cantabres. Malgré l'ancienneté de la plupart des recherches, la succession des industries est en accord avec celle de la Riera, ce qui nous permet de confirmer la validité de ce nouveau modèle reconnaissant l'existence du Badegoulien cantabrique, dans un même environnement climatique froid et humide. Enfin, les auteurs discutent du problème des transitions Solutréen/Badegoulien et Badegoulien/Magdalénien dans les Cantabres et de la structure interne du Badegoulien cantabrique.
\end{abstract}

\begin{abstract}
A new interpretation of the sequence of La Riera (Asturias) is proposed, based on a multidisciplinary analysis including the palaeoenvironment, the patterns of lithic raw material procurement, the knapping process and the lithic and bone industries. After a short summary of the model established by L.G. Straus, the review of the palaeoclimatological framework underlines a very cold contextwith some more humid episodes - for the whole stratigraphy, except for the top of the sequence corresponding to the end of the Würmian ice age interstadial warming ("Bølling/Allerød"). Analysis of the raw materials and the knapping process shows a mainly local subsistence economy for the end of the Solutrean and the Badegoulian, coinciding with the Last Glacial Maximum, in contrast with the beginning of the Solutrean and the Magdalenian where circulation along the Cantabrian coast as far as the south of the Aquitanian basin is conceivable. The results of the statistical processing of the stone industries reveal the presence of several industrial facies in stratigraphic sequence, including a new entity: the Cantabrian Badegoulian. Typological analyses
\end{abstract}


demonstrate the existence of continuities and discontinuities between these differents facies. The bone industries underline strong convergences between the Solutrean and the Badegoulian. This structuration is then compared with other data available for the period between 22000 BP and 16000 BP in Cantabria. In spite of the age of most research, the sequence of industries fits well with that of La Riera, which allows us to confirm the validity of this new model recognizing the existence of the Cantabrian Badegoulian, in the same cold and damp climatic environment ("Lascaux"). Finally, the authors discuss the problem of the Solutrean/Badegoulian and Badegoulian/Magdalenian transitions in Cantabria and of the internal structure of the Cantabrian Badegoulian.

\section{INTRODUCTION}

Le site de la Riera dans les Asturies (Espagne), fouillé par L.G. Straus, constitue un gisement de référence pour la connaissance du Solutréen cantabrique. Les données issues de la publication de cette étude pluridisciplinaire (Straus et Clark éd., 1986), englobant une analyse détaillée du contexte paléoclimatique situé entre 21000 B.P. et 8000 B.P., nous permettent aujourd'hui de réviser la séquence chronostratigraphique de ce site et de considérer la question de l'existence d'un "Badegoulien cantabrique", (Corchon-Rodriguez, 1994, p. 134 ; Rasilla-Vives, 1994 ; Utrilla-Miranda, 1986, note 13).

Après un bref historique sur le gisement et le modèle d'occupation proposé par L.G. Straus, le cadre paléoclimatique de cette séquence est discuté et corrigé. Les résultats des traitements statistiques concernant les matières premières, le débitage, les outillages lithiques et osseux sont ensuite détaillés par faciès industriels, dont les caractéristiques sont alors présentées. Est alors proposée une nouvelle interprétation de la séquence de la Riera. Enfin, une synthèse des industries situées entre 22000 B.P. et 16000 B.P. dans les Cantabres permet d'étendre à cette région un modèle de peuplement au cours du pléniglaciaire du Würm récent.

\section{LA SÉQUENCE DE LA RIERA D'APRÈS L.G. STRAUS}

\section{Historique des fouilles}

Le gisement de la Riera, situé à mi-chemin d'Oviedo et de Santander dans les Asturies (Espagne), se trouve dans la vallée du Rio Calabres, à proximité de la ville de Llanes. Il s'agit d'une petite cavité située sur les contreforts de la Sierra de Cuera, à environ $1,5 \mathrm{~km}$ de la mer. À l'époque préhistorique, elle se trouvait à égale distance de la mer et du sommet englacé du Picos de Europa $(2500-2600 \mathrm{~m})$.

Découvert par R. Duque de Estrada y Martinez de Morentin et Condé de la Vega del Sella, des premiers sondages furent entrepris vers 1914, où l'on y reconnût une longue séquence de l'Aurignacien, du Solutréen, du Magdalénien, de l'Azilien et de l'Asturien. Des fouilles systématiques furent conduites en 1917-1918 par Condé de la Vega del Sella, (Vega del Sella, 1930). Une fouille de contrôle de G.A. Clark en 1969, puis un sondage de J.M. Gomez-Tabanera et M. Perez en 1972, ont conduit L.G. Straus et son équipe à entreprendre plusieurs campagnes de fouilles entre 1976 et 1979 , et à préciser la stratigraphie et les industries préhistoriques associées, (Straus et Clark, 1986a).

\section{Le contexte paléoclimatique et les datations absolues}

À partir des études du paléoenvironnement (Altuna, 1986; Laville, 1986 ; Arl. Leroi-Gourhan, 1986), L.G. Straus propose un tableau de la séquence chronoclimatique de la Riera, (Straus, 1986a et b). La base de la couche 1 est caractérisée par un climat froid, à hivers subartiques et étés plus humides. Le sommet de la couche 1 et la couche $2+3$ se situent en environnement plus modéré et très humide ("interstade Würm III/IV"). Les couches 4 à 8 voient le retour d'un froid beaucoup plus sec correspondant aux conditions les plus sévères du pléniglaciaire du Würm récent. Les couches 9 à 18 révèlent l'existence d'une pulsation tempérée et humide, voyant une reforestation (limitée) de la région ("oscillation de Lascaux"). Les couches 19, 20 et peut-être 21 se situent dans un épisode de nouveau froid et sec, qui marque la fin des conditions glaciaires en Espagne. Le contexte des couches 21 à 23 pose de nombreux problèmes, du fait de contradictions entre les résultats de la sédimentologie et de la palynologie. Sur la base des datations absolues, L.G. Straus place ces niveaux à l'interface Dryas II/Allerød. La couche 24 se caractérise par un environnement relativement froid et humide. Avec les niveaux 25 et 26, on assiste à l'installation d'un climat tempéré et humide, correspondant à la fin de l'oscillation d'Allerød. Cette amélioration est ensuite brièvement interrompue dans les couches 27 et 28, marquées par les conditions plus froides du Dryas III. Enfin, les couches 29 et 30 représentent les dernières manifestations du tardiglaciaire (Pré-Boréal et Boréal).

Une analyse des nombreuses datations absolues conduit L.G. Straus à proposer un tableau de la chronologie de la Riera, (Straus, 1986a). La couche 1 se situerait à environ $20000-20500$ B.P., les couches 4 à 10 entre environ 20500 B.P. et $19000-19500$ B.P. et la 


\begin{tabular}{|c|c|c|c|c|c|c|c|c|c|c|c|c|}
\hline \multirow{2}{*}{ 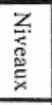 } & \multicolumn{3}{|c|}{ SÉDIMENTOLOGIE } & \multicolumn{4}{|c|}{ PALYNOLOGIE } & \multirow{2}{*}{ 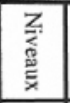 } & \multicolumn{2}{|c|}{ FAUNE } & \multirow{2}{*}{$\begin{array}{c}\text { CHRONOLOGIE } \\
\text { L.G. STRAUS }\end{array}$} & \multirow{2}{*}{ INDUSTRIES } \\
\hline & Climat & Épisode & Dates BP & Climat & Végétation & Épisode & Dates BP & & Indicateurs & $\%$ Cerf & & \\
\hline 30 & $\begin{array}{c}\text { Tempéré } \\
\text { Très Humide } \\
\end{array}$ & Borćal & \multirow{5}{*}{$\begin{array}{c}8650 \mathrm{BP} \\
10150 \mathrm{BP} \\
10750 \mathrm{BP}\end{array}$} & Humide & \begin{tabular}{|c|}
$\begin{array}{c}\text { tilleul, noyer } \\
\text { aulne, noisetier }\end{array}$ \\
\end{tabular} & \multirow[t]{2}{*}{ Boréal } & \multirow{3}{*}{$\begin{array}{c}8650 \mathrm{BP} \\
10750 \mathrm{BP}\end{array}$} & & & & \multirow{2}{*}{$\begin{array}{l}\text { Boréal } \\
\text { (et Pré-Boréal ?) }\end{array}$} & \\
\hline 29 & Frais, Humide & Pré-Boréal & & \multirow[b]{2}{*}{$\begin{array}{l}\text { Plus } \\
\text { Chaud }\end{array}$} & \multirow{5}{*}{\begin{tabular}{|c|} 
aulne \\
saule \\
orme \\
noyer \\
noisetier \\
chêne
\end{tabular}} & & & 29 & & 84,3 & & Asturien \\
\hline $\begin{array}{l}28 \\
27\end{array}$ & $\begin{array}{c}\text { Plus Froid } \\
\text { Mais Humide }\end{array}$ & Dryas III & & & & \multirow{4}{*}{ Allerød } & & $\begin{array}{l}28 \\
27\end{array}$ & $\begin{array}{l}\text { Capreolus, Sus } \\
\text { Capreolus, Sus }\end{array}$ & $\begin{array}{l}70,0 \\
76,0\end{array}$ & Dryas III & Azilien \\
\hline & Erosion & & & & & & \multirow{6}{*}{$11750 \mathrm{BP}$} & & & & \multirow{4}{*}{ Allerød } & \multirow{9}{*}{ Magdalénien } \\
\hline $\begin{array}{l}26 \\
25\end{array}$ & $\begin{array}{l}\text { Tempéré } \\
\text { Très Humide }\end{array}$ & Allerød & & Humide & & & & $\begin{array}{l}26 \\
25\end{array}$ & Capreolus, Sus & $\begin{array}{l}68,1 \\
74,0\end{array}$ & & \\
\hline 24 & Froid, Humide & Dryas II & \multirow{2}{*}{$\begin{array}{l}11750 \text { BP } \\
12250 \text { BP } \\
14750 \text { BP }\end{array}$} & & & & & 24 & Rangifer & 52,2 & & \\
\hline \multicolumn{2}{|c|}{ Erosion-Hiatus } & Bølling & & & \multirow{3}{*}{ genevrier } & \multirow{3}{*}{ Dryas II } & & \multirow{3}{*}{$\begin{array}{l}23 \\
22 \\
21\end{array}$} & \multirow{3}{*}{$\begin{array}{c}\text { Sus } \\
\text { Rangifer }\end{array}$} & \multirow{3}{*}{84,3} & & \\
\hline \begin{tabular}{l|}
23 \\
22 \\
\end{tabular} & $\begin{array}{c}\text { Tempéré } \\
\text { Très Humide }\end{array}$ & Angles & \multirow{5}{*}{$\begin{array}{l}15350 \mathrm{BP} \\
16450 \mathrm{BP}\end{array}$} & Froid & & & & & & & & \\
\hline \multicolumn{2}{|c|}{ Cryoturbation } & \multirow{3}{*}{$\begin{array}{c}\text { Inter } \\
\text { Lascaux / } \\
\text { Angles }\end{array}$} & & & & & & & & & \multirow{2}{*}{$\begin{array}{l}\text { Dryas I-II ? } \\
\text { hiatus }\end{array}$} & \\
\hline $\begin{array}{l}21 \\
20\end{array}$ & $\begin{array}{l}\text { Froid, } \\
\text { Progressivement }\end{array}$ & & & hiatus & & & \multirow[t]{2}{*}{$12250 \mathrm{BP}$} & 20 & & 81,1 & & \\
\hline 19 & Plus Sec & & & Plus Froid & végétation de & ? & & 19 & & 77,6 & & \\
\hline $\begin{array}{l}18 \\
17\end{array}$ & Moins Tempéré & & & Plus Sec & steppe & & & 18 & & 82,0 & $\begin{array}{c}\text { Inter } \\
\text { Lascaux / }\end{array}$ & \\
\hline 16 & Humide & & & & & & $16450 \mathrm{BP}$ & 16 & Bison priscus & $\begin{array}{l}80,7 \\
80,0\end{array}$ & & \\
\hline 15 & (optimum) & & & & & & & 15 & & 80,0 & & \\
\hline $\begin{array}{l}14 \\
13\end{array}$ & Encore Plus & Lascaux & & Tempéré & $\begin{array}{l}\text { orme } \\
\text { noisetier }\end{array}$ & & & $\begin{array}{l}14 \\
13\end{array}$ & & $\begin{array}{l}77,4 \\
76,6\end{array}$ & & \\
\hline 12 & $\begin{array}{l}\text { Tempéré et } \\
\text { Humide }\end{array}$ & & & & $\begin{array}{l}\text { noisetier } \\
\text { chêne }\end{array}$ & Lascaux & & 12 & & 82,6 & Lascaux & \\
\hline 11 & & & & Humide & bouleau & & & 11 & & 90,4 & & \\
\hline $\begin{array}{c}10 \\
9\end{array}$ & $\begin{array}{l}\text { Tempéré } \\
\text { Humide }\end{array}$ & & & & aulne & & & $\begin{array}{c}10 \\
9\end{array}$ & Capreolus & $\begin{array}{l}83,0 \\
80,7\end{array}$ & & Solutréen \\
\hline 8 & & & $17950 \mathrm{BP}$ & & & & $17950 \mathrm{BP}$ & 8 & & 70,2 & & \\
\hline $\begin{array}{l}7 \\
6\end{array}$ & Froid & $\begin{array}{c}\text { Inter } \\
\text { Laugerie / }\end{array}$ & & Froid, Sec & $\begin{array}{l}\text { lande } \\
\text { à composées }\end{array}$ & Inter & & $\begin{array}{l}7 \\
6\end{array}$ & Microtus & $\begin{array}{l}71,2 \\
57,1\end{array}$ & & \\
\hline 5 & FTord & Lascaux & & Perturbé & - & Laugerie / & & 5 & & 43,5 & $\begin{array}{l}\text { Inter } \\
\text { Laugerie/ }\end{array}$ & \\
\hline 4 & & & $18950 \mathrm{BP}$ & Froid & lande & Lascaux & & 4 & & 32,1 & Lascaux & \\
\hline 3 & Tempéré & & & Humide & & & $18950 \mathrm{BP}$ & 3 & & 17,4 & & \\
\hline 2 & Humide & Laugerie & & & pin & & & 2 & & & & \\
\hline 1 sup & $\begin{array}{l}\text { Erosion } \\
\text { Très Humide }\end{array}$ & & & Tempéré & $\begin{array}{l}\text { noisetier } \\
\text { chêne }\end{array}$ & Laugerie & & 1 sup & Bos & 44,3 & Laugeric & Aurignacien? \\
\hline 1 inf & Froid & Inter $\mathrm{T} / \mathrm{L}$ & $19950 \mathrm{BP}$ & $=$ & - & $=$ & $19950 \mathrm{BP}$ & $1 \mathrm{inf}$ & us & & & \\
\hline
\end{tabular}

Fig. 1 - Séquence chronostratigraphique et culturelle de la Riera d'après L.G. Straus (Straus, 1986a, p. 22-23).

couche 17 à environ 17000 B.P., ce qui placerait la séquence des couches 12 à 16 entre environ 19000 B.P. et 17000 B.P. Les couches 19 et peut-être 20 base se situeraient à environ 16000 B.P., les couches 23 à 27 entre 12000 B.P. et 10500 B.P. et enfin la couche 29 entre environ 9000 B.P. et 7000 B.P.

\section{Les industries préhistoriques}

Sur la base de l'abondance des grattoirs, dont un exemplaire à museau caréné, des lamelles Dufour et de l'absence des lamelles à dos et des outils solutréens, L.G. Straus attribue la couche 1 à l'Aurignacien, (Straus, 1986c). L'industrie se caractérise par l'abondance des encoches et denticulés, l'équilibre entre les grattoirs et les burins et l'absence des lamelles à dos et des outils solutréens.

La présence de pièces solutréennes, en très faible nombre dans les niveaux supérieurs (couches 9 à 18, sauf la couche 10), incite L.G. Straus à placer les couches $2+3$ à 18 (Straus, 1986a, p. 23) dans le Solutréen. La variabilité typologique de l'ensemble est interprétée comme la conséquence d'une modification dans les stratégies de chasse: l'importance des grattoirs, des encoches et denticulés et la rareté des lamelles à dos étant corrélée à la fréquence du cerf, l'importance des lamelles à dos et des outils solutréens étant corrélée à la fréquence du bouquetin et du chamois.
Sur la base de l'absence conjointe des harpons et des outils solutréens et de la présence de sagaies de section quadrangulaire, L.G. Straus attribue les couches 18 (Straus, 1986c, p. 230) à 20 au Magdalénien inférieur. L'industrie se caractérise essentiellement par le foisonnement des lamelles à dos, la rareté des encoches et denticulés et l'équivalence entre les grattoirs et les burins. À partir des datations absolues, L.G. Straus attribue les couches 21 à 23 et 24 au Magdalénien supérieur. Pour la couche 24 (les niveaux 21 à 23 étant pauvres), l'industrie se caractérise par un équilibre typologique similaire à celui du Magdalénien inférieur, hormis une augmentation des grattoirs, dont des formes courtes sur éclat, et l'apparition de quelques pointes aziliennes.

L'appauvrissement en lamelles à dos, le développement des grattoirs courts, des pointes aziliennes et la présence d'un exemplaire de harpon plat (Azilien) incitent L.G. Straus à placer les couches 26 à 28 dans 1'Azilien. L'industrie se caractérise par la supériorité des grattoirs sur les burins, le développement variable des lamelles à dos et la présence significative des grattoirs courts et des pointes aziliennes. Il développe par ailleurs une hypothèse selon laquelle l'Azilien et l'Asturien (couche 29) ne seraient que deux faciès fonctionnels d'une même population, (Straus et Clarke, 1986c, p. $380-381$ ).

L'interprétation de L.G. Straus de la séquence chronoclimatique et culturelle de la Riera (Straus, 1986a, p. 22-23) est présentée sur la figure 1 . 


\section{UNE RÉVISION DE LA SÉQUENCE DE LA RIERA}

\section{Le contexte paléoclimatique}

\section{La sédimentologie}

L'étude sédimentologique, effectuée par H. Laville (Laville, 1986), a été réalisée à partir de trois séries de prélèvements, nommées ici section A (carrés E5 à E8 et F4), section B (carré E7) et section C, la plus en avant de la grotte, (carrés F10 et G10).

Une analyse statistique élémentaire, conduite sur les valeurs moyennes calculées à partir de ces trois séries, nous amène à dégager des caractéristiques sédimentologiques globales (fig. 2A). L'ensemble de base (couches 1 à 8) voit l'abondance des éboulis fragmentés et des cailloux gélivés. L'absence des cailloux gélivés et la plus forte représentation des concrétions calcaires dans le niveau de base semble indiquer un effet de substratum, sur lequel se sont accumulées les infiltrations et les percolations. Le deuxième ensemble (couches 9 à 12) se caractérise par la réduction progressive des éboulis fragmentés, une plus faible proportion de cailloux gélivés et l'augmentation sensible des concrétions calcaires, ce qui indique une circulation d'eau plus intense dans les sédiments. De la couche 14 à la couche 23 , les dépôts, plus fins que dans les niveaux sousjacents, sont associés à de rares cailloux gélivés et concrétions calcaires. Enfin, le sommet de la séquence (couches 24 à 29) voit la disparition définitive des cailloux gélivés et la très forte progression des concrétions calcaires, signes d'une nette augmentation de la circulation d'eau.

Une analyse, menée sur l'ensemble des prélèvements des trois secteurs, permet d'appréhender les variations latérales de ces faciès sédimentologiques (fig. 2B). Ainsi, la couche 1 , très homogène, traduit un effet de substratum'. Les couches 2 à 12 du Proto-Solutréen (couches 2 et 3), du Solutréen récent (couches 4 à 7) et du Badegoulien cantabrique (couches 8 à 12) montrent des variations latérales faibles, qui restent cependant dans les limites de définition des faciès sédimentaires. À l'inverse, les couches 14 à 23 de la fin du Badegoulien cantabrique (couches 14 à 16), du Magdalénien inférieur cantabrique (couches 17 à 20) et du Magdalénien (supérieur? : couches 21 à 23) montrent des variations latérales importantes, indiquant soit des perturbations d'origine géologique, soit des problèmes de raccords stratigraphiques entre secteurs. Ce phénomène, particulièrement sensible pour les couches 14 à 17,23 et, dans une moindre mesure, 18 , peuvent induire des mélanges plus ou moins importants dans les industries préhistoriques. Les couches 24 à 28 de l'Azilien montrent enfin des variations latérales faibles. En conclusion, l'importance des variations latérales dans les faciès sédimentologiques des couches 14 à 17 soulève un doute quant à l'intégrité des industries préhistoriques associées, en particulier en ce qui concerne la représentativité des lamelles à dos, et ne permet pas de conclure quant à l'existence d'un éventuel faciès de transition entre le Badegoulien et le Magdalénien inférieur cantabrique (couches 15 et 16).
L'analyse factorielle des correspondances permet de mettre en évidence deux axes interprétables en tant que gradients de température et d'humidité. Le climat reste froid sur l'ensemble de la séquence, jusqu'aux niveaux 23 et 24 marquant le début de l'oscillation d'Allerød, avec de fortes variations d'humidité dans les ensembles 2 à 8,9 à 12 et 24 à 28 , correspondant aux épisodes de "Laugerie", "Lascaux" et "Allerød". L'existence de lacunes importantes se confirme, entre les couches 1 et 2 (correspondant à l'absence du Solutréen ancien), 8 et 9 (Dryas I inter Laugerie/Lascaux), dans la couche 20 (fin du Dryas I/Pré-Bølling : cf. infra) et 28 et 29 (absence du Dryas III). En conclusion, l'analyse sédimentologique permet de distinguer seulement trois épisodes pendant lesquels la circulation d'eau a été intense dans les dépôts contemporains: pour les couches 4 à 8 ("épisode de Laugerie"), pour les couches 9 à 12 (“épisode de Lascaux"), et pour les couches 24 à 28 (Allerød).

\section{La paléontologie}

L'étude paléontologique a été réalisée par J. Altuna, (Altuna, 1986). La figure 3 présente les variations quantitatives des principales espèces rencontrées sur le site de la Riera.

Les couches 1 à 3 se caractérisent par l'abondance du bison et du cheval, animaux de steppe, des bouquetins, des chamois et la rareté du cerf. Dans les couches 4 à 7 , on assiste à la disparition du bison et du cheval et à un équilibre entre le cerf et les animaux de montagne (bouquetin, chamois), ce qui suggère un approvisionnement préférentiellement local, phénomène qui s'amplifiera de la couche 8 à la couche 23. À partir de la couche 24 , et jusqu'à la couche 29 , le développement du chevreuil et du sanglier, espèces forestières apparues dès la couche $21-23$, traduit une très nette amélioration du climat.

En conclusion, l'étude de la faune nous incite à envisager de profondes modifications dans la stratégie d'approvisionnement des chasseurs-cueilleurs de la Riera, entre des approvisionnements locaux et dans des déplacements jusque dans le sud du bassin aquitain.

\section{La palynologie}

L'étude palynologique a été réalisée par Arl. LeroiGourhan, (Arl. Leroi-Gourhan, 1986).

Une analyse factorielle des correspondances puis une classification automatique effectuée sur les 5 premières coordonnées factorielles mettent en évidence une zonation pollinique en 3 classes (fig. 4A) :

- un cortège à Éricacées et Carduacées (couches 4 à 16 inf.), divisée, sur les axes plus lointains, en 2 sousensembles : couches 4 à 8 d'une part, couches 9 à 16 inf. d'autre part. Pour Arl. Leroi-Gourhan, il s'agit d'une végétation de lande atlantique;

- un cortège ${ }^{2}$ à Composées Liguliflores, Anthémidées et Carduacées (couches 16 sup. à 23/24), contenant en outre de nombreux Juniperus dans les couches 22 et 23. Pour Arl Leroi-Gourhan, il s'agit d'une végétation de steppe ;

- une boulaie à Filicales (couches 24 à 29). 

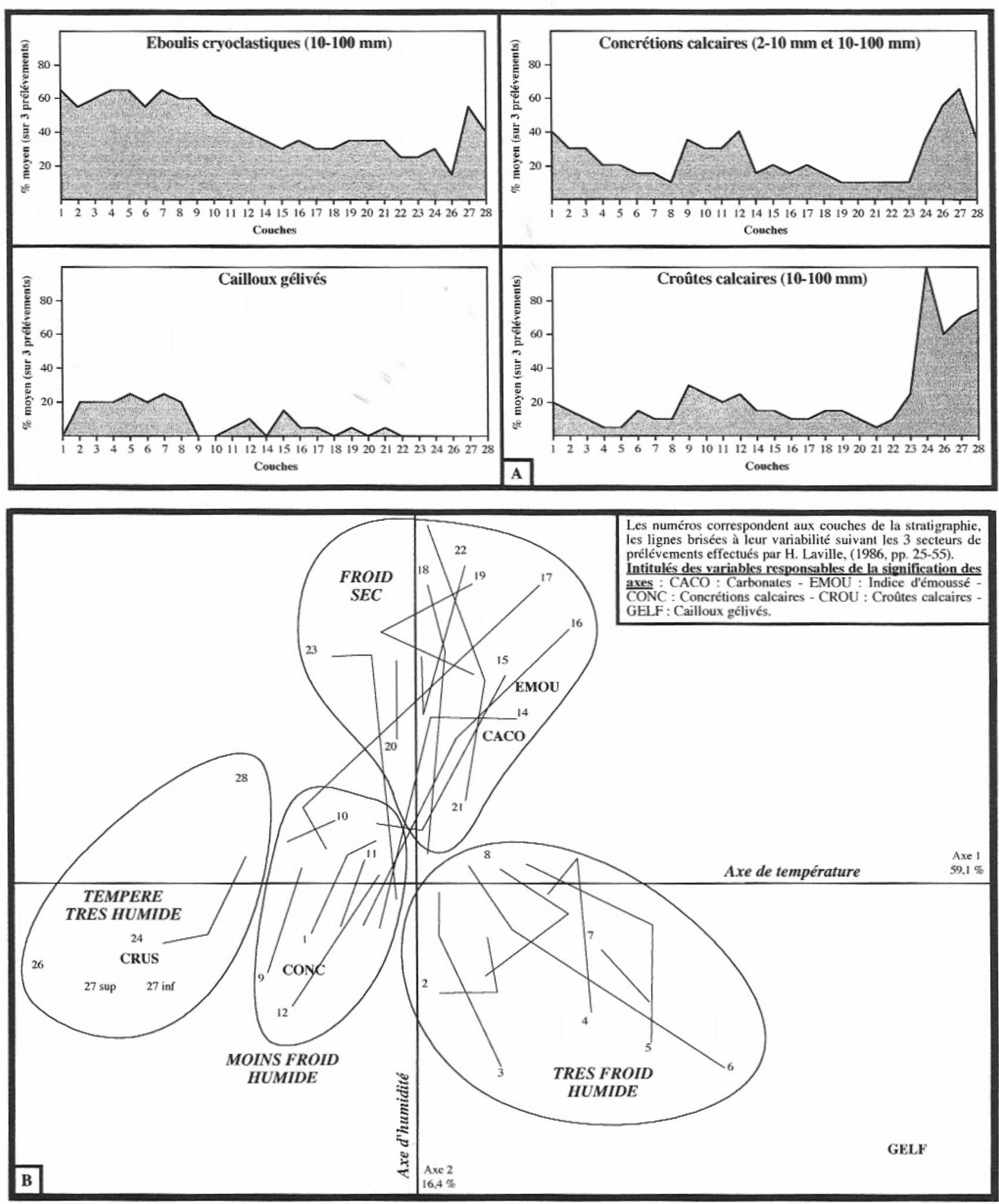

Fig. 2 - Étude sédimentologique des dépôts de la séquence de la Riera. 2A : variations des caractéristiques sédimentologiques globales en stratigraphie : valeurs moyennes calculées sur trois secteurs de prélèvements (Laville, 1986); 2B : variations latérales des faciès sédimentologiques : plan factoriel 1-2 de l'analyse factorielle des correspondances.

Le premier ensemble (couches 4 à 16 inf.) se caractérise par un faible taux de pollens d'arbres, la rareté des Composées Liguliflores et le fort développement des Éricacées et, dans une moindre mesure, des Carduacées.
Le deuxième ensemble (couches 16 sup. à 23/24) se caractérise (note 2) par une légère augmentation du taux de pollens d'arbres, la réduction massive des Éricacées et le développement des Composées Liguliflores, des 
Anthémidées et des Carduacées. Dans les couches 22 et 23 , on assiste en outre à l'expansion des Juniperus, espèce caractéristique de la reconquête végétale du début de l'oscillation de Bølling, (Leroyer, communication personnelle).
Le troisième ensemble enfin (couches 24 à 29) voit la forte progression du taux de pollens d'arbres, essentiellement des bouleaux, et des Filicales, apparues dans les couches 22 et 23 , la réduction des Composées Liguliflores et la quasi disparition des Juniperus. I1

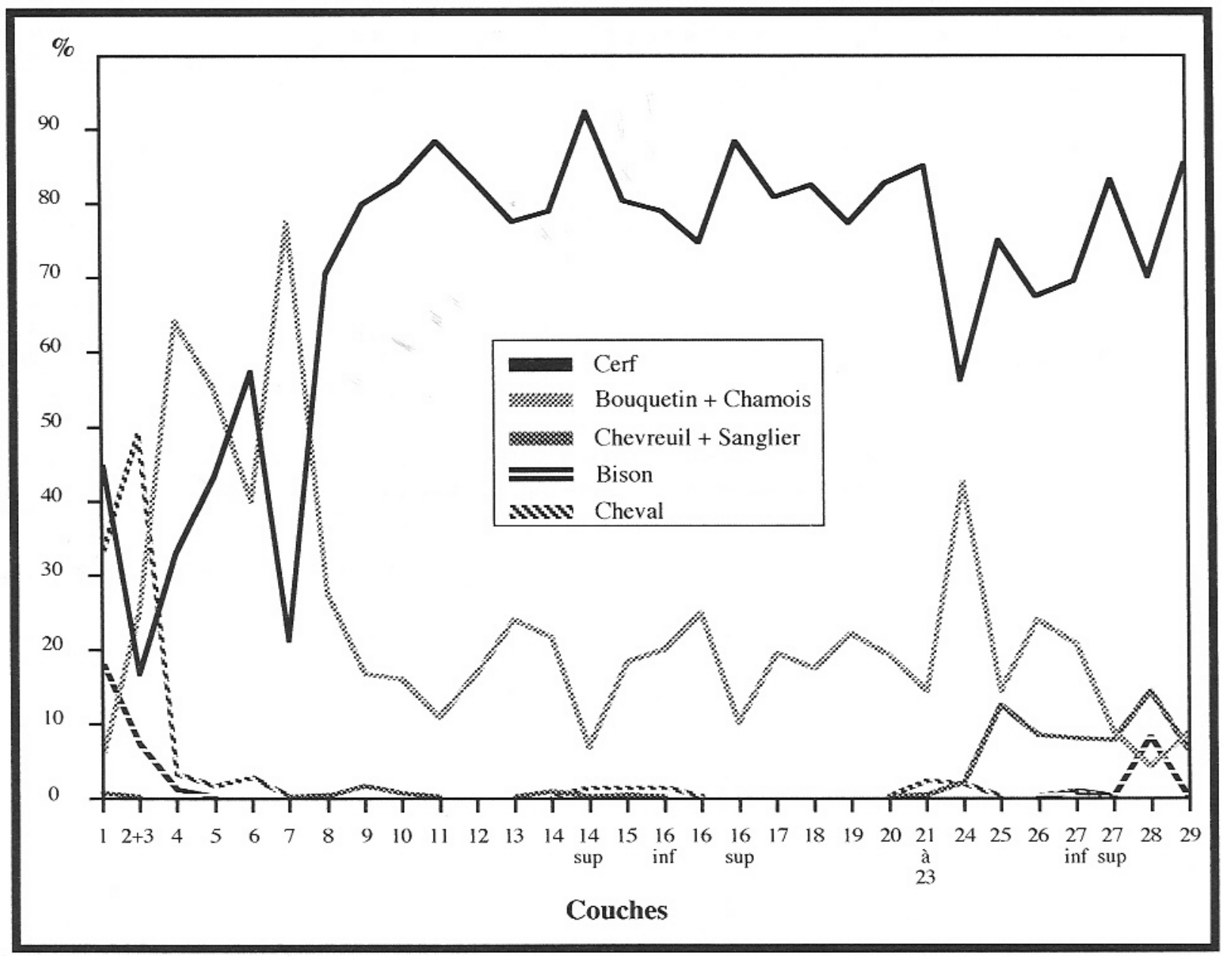

Fig. 3 - Variations des espèces faunistiques dans la séquence de la Riera.
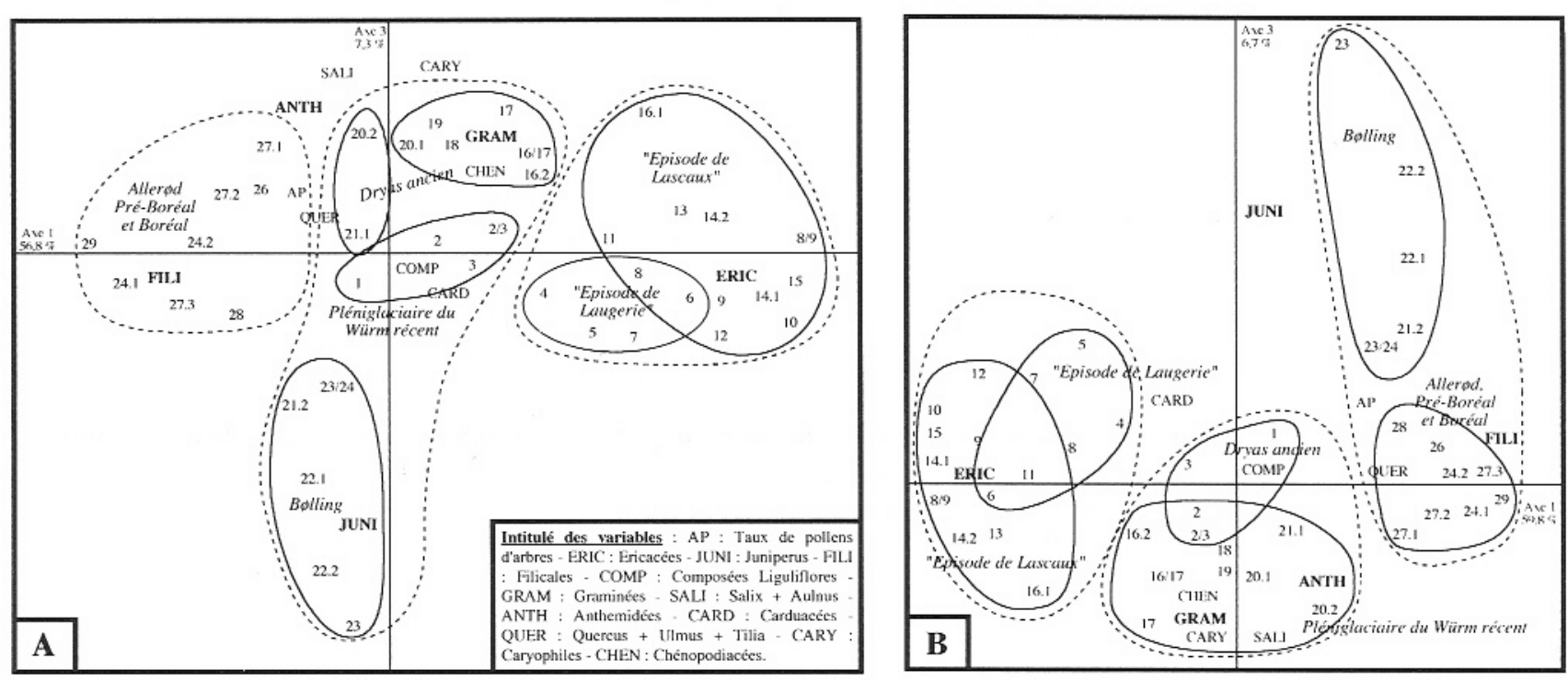

Fig. 4-Étude palynologique de la séquence de la Riera. 4A : plan factoriel 1-3 de l'analyse factorielle des correspondances, les Juniperus étant exclus du taux de pollens d'arbres (les variables responsables de la signification des axes sont figurées en gras); $4 \mathbf{B}:$ plan factoriel 1-3 de 1'analyse factorielle des correspondances, les Juniperus étant inclus dans le taux de pollens d'arbres (les variables responsables de la signification des axes sont figurées en gras). 
correspond à une végétation de "boulaie à Filicales" caractéristique de l'oscillation d'Allerød.

Cette analyse, dans laquelle les Juniperus n'ont pas été comptés dans le pourcentage de pollens d'arbres, confirme une végétation ouverte (et froide) pour la base de la séquence, variant simplement suivant le degré d'humidité (steppe ou lande) ${ }^{3}$. L'exclusion des Juniperus du taux de pollens d'arbres renforce artificiellement le caractère ouvert et froid de la végétation des couches 22 et 23 , par une sous-estimation de la valeur de AP (Arborean Pollens) et une surestimation des taxons herbacés steppiques (Composées Liguliflores).

Une analyse factorielle des correspondances, intégrant les Juniperus dans le taux de pollens d'arbres, puis une classification automatique, mettent en évidence une nouvelle zonation pollinique en 3 classes (fig. 4B) :

- un cortège à Éricacées et Carduacées (couches 4 à 16 inf.);

- un cortège à Composées Liguliflores, Anthémidées et Carduacées (couches 16 sup. à 21 inf.);

- un cortège à nombreux pollens d'arbres, divisé, sur les axes factoriels 3 et 4, en deux sous-ensembles : à Juniperus (couches 21 sup. à 23) ; à bouleaux et Filicales (couches 24 à 29).

Cette seconde analyse, qui se distingue de la première essentiellement au niveau de l'attribution des couches 21 sup. à 23 , confirme une dynamique de reforestation du paysage en deux étapes: l'apparition de massifs denses de Juniperus sur un substrat de lande ou de steppe, au cours de l'oscillation de Bølling, puis l'établissement d'une boulaie à Filicales, au cours de l'oscillation d'Allerød.

En conclusion, il se confirme donc la présence d'une végétation très ouverte pour la base de la séquence (lande ou steppe: couches 1 à 21 inf.), puis une reforestation progressive, et en deux temps, du paysage lors d'une oscillation tardiglaciaire Bølling/Allerød (couches 21 sup. à 28), le Dryas II n'étant alors qu'une réminiscence dans cette dynamique conduisant à l'holocène.

\section{Synthèse}

À partir d'une analyse quantitative réalisée sur l'ensemble de ces données (sédimentologie, paléontologie et palynologie), le contexte paléoclimatique de la séquence de la Riera peut être discuté et précisé.

Le premier axe factoriel (41,9\% d'inertie) traduit un gradient de température, en mettant clairement en évidence une opposition entre un épisode de lande à Éricacées et cailloux gélivés (couches 4 à 16) et un épisode de boulaie à Filicales (couches 24 à 29). Le deuxième axe (19,6\% d'inertie) souligne l'originalité des niveaux 1 et $2+3$, dont la faune est composée de chevaux et de bovinés. Le troisième axe $(11,4 \%$ d'inertie) oppose des niveaux à faune dominée par le cerf ou le cheval et/ou à Éricacées à des niveaux à cailloux gélivés et/ou à faune à bouquetins et chamois (couches 4 à 8). Le quatrième axe (10,0\% d'inertie) traduit l'originalité des couches 17 à 20 et surtout 21 à 23, à Composées Liguliflores, Anthémidées, Juniperus et faune dominée par le cerf à des ensembles à Éricacées ou à Filicales. Le cinquième axe enfin (6,2\% d'inertie) confirme le développement massif des Juniperus dans les couches 21 à 23. Une classification automatique, effectuée sur ces 5 premières coordonnées factorielles, met en évidence une partition en 7 classes (fig. 5) :

- couches 1 et $2+3$;

- couches 4 à 7 ;

- couches 8 à 16 ;

- couches 17 à 20 ;

- couches 21-23;

- couches 24 à 28 ;

- couches 29 (et 30 ?).

Les analyses factorielles des correspondances partielles de trois disciplines de la paléoclimatologie (sédimentologie, paléontologie et palynologie) mettent en évidence, sur les deux premiers axes, un gradient de température et un gradient d'humidité qui se retrouvent sur l'analyse factorielle des correspondances globale (les Juniperus ont été intégrés dans le taux de pollens d'arbres), sur les axes 1 et 4, et qui structurent le paléoenvironnement de la séquence stratigraphique de la Riera de la façon suivante :

- couches 1 à 3 : Fin du pléniglaciaire du Würm récent, froid et sec;

- lacune;

- couches 4 à 7 : “Épisode de Laugerie”, très froid et humide ;

- lacune;

- couches 8 à 16: “Épisode de Lascaux", froid et humide ;

- perturbation stratigraphique;

- couches 17 à 20 : Dryas I, froid et sec ;

- lacune ${ }^{4}$;

- couches 21 à 23 : Fin du Dryas I (couche 21 inf.) et oscillation de Bølling (couches 21 sup. à 23), marquée par une première reconquête de la forêt (Juniperus) sur une végétation de steppe;

- couches 24 à 28: Oscillation d'Allerød, tempéré et humide;

- lacune;

- couche 29 (et 30 ?) : Holocène (Pré-Boréal et Boréal), tempéré et humide.

\section{Les analyses des industries lithiques}

\section{Les matières premières et le débitage}

\section{- Les matières premières}

Les matières premières utilisées à la Riera (Straus et Clark, 1986b ; Straus et al., 1986) sont constituées de 28 variétés, groupées en 3 catégories : les silex (types A, B, C, D, E, F, G, H, O, Q, R, U, W, AA et BB), les quartzites (types I, J, K, L, P, S et V) et les “divers" (types T, $\mathrm{N}, \mathrm{X}, \mathrm{Y}, \mathrm{Z}$ et $\mathrm{M})$, ce dernier $(\mathrm{M}=$ " sandstone ") n'étant pas pris en compte ici.

Le tableau ci-dessous (tabl. 1) montre que certaines variétés sont toujours rares dans la séquence, et que d'autres présentent un développement singulier : silex de type E, F, G, H, O, R, U, AA et BB, quartzites de 

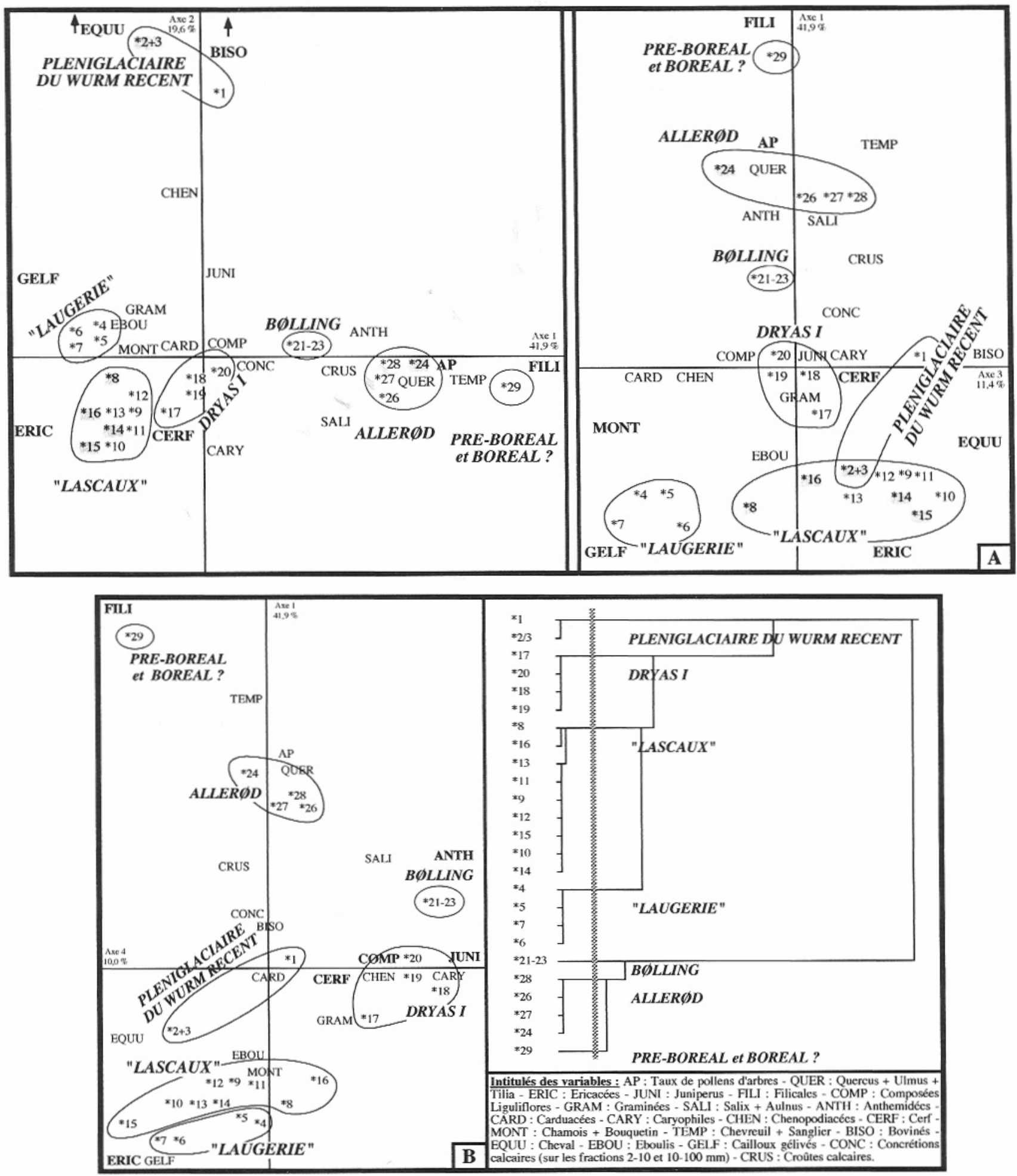

Fig. 5-Paléoclimatologie de la séquence de la Riera. 5A : plans factoriels 1-2 et 1-3 de l'analyse factorielle des correspondances globale (sédimentologie, palynologie et paléontologie) (les variables responsables de la signification des axes sont figurées en gras); 5 B : plan factoriel 1-4 de l'analyse factorielle des correspondances globale (sédimentologie, palynologie et paléontologie) et arbre de la classification automatique (les variables responsables de la signification des axes sont figurées en gras).

type $\mathrm{K}, \mathrm{P}, \mathrm{S}, \mathrm{V}$ et, dans une moindre mesure $\mathrm{L}$, et "divers" (type T, N, X, Y et Z).

De façon à minimiser ces variabilités, nous avons procédé à des agrégations de types, à partir des descriptions macro- et microscopiques des matériaux. Nous avons ainsi défini 12 catégories: silex de type S1 (A), S2
$(\mathrm{B}+\mathrm{D}+\mathrm{R}), \mathrm{S} 3(\mathrm{C}+\mathrm{F}+\mathrm{G}), \mathrm{S} 4(\mathrm{H}+\mathrm{O}+\mathrm{BB}), \mathrm{S} 5$ $(\mathrm{U}+\mathrm{E}), \mathrm{S} 6(\mathrm{Q}+\mathrm{W}+\mathrm{AA})$, total des silex ; quartzites de type QI (I), QJ (J), QDV $(\mathrm{K}+\mathrm{L}+\mathrm{P}+\mathrm{S}+\mathrm{V})$, total des quartzites; "divers" $(\mathrm{M}+\mathrm{N}+\mathrm{T}+\mathrm{X}+\mathrm{Y}+\mathrm{Z})$. À cette typologie, nous avons inclus les pourcentages d'outils et de déchets de débitage en silex et en quartzite. 
Une analyse factorielle des correspondances et une classification automatique, dont les résultats sont présentés sur la figure 6 , mettent en évidence une structure de partition en 4 classes, liée aux "faciès archéologiques” ( $c f$. infra l'étude des outillages lithiques) :

- Ie début du Solutréen récent (couches 2 à 5) est caractérisé par l'importance des silex S2, S6 et, dans une moindre mesure, S4, et des “divers”. À l'inverse, les quartzites y sont très rares. L'abondance du silex se retrouve tant pour les outils que pour les déchets de débitage ;

- la fin du Solutréen récent et le Badegoulien cantabrique (couches 6 à 16) ${ }^{5}$ sont caractérisés par le développement massif des quartzites QI et QJ et la forte réduction des silex. Toutefois, le choix des silex et des quartzites s'équilibrent pour le façonnage des outils ; - Ie Magdalénien inférieur cantabrique (couches 17 à 20) est caractérisé par l'importance des silex S1, S3 et, dans une moindre mesure, S2, et des quartzites QJ et QDV. La supériorité du choix des silex sur les quartzites est beaucoup plus manifeste pour le façonnage des outils que pour le débitage. Les niveaux magdaléniens (supérieur?: couches $21-23$ et 24 ) sont très pauvres ;

- l'Azilien enfin (couches 26 à 28) est caractérisé par l'importance du silex S1, des quartzites QI et QDV et des "divers". Si le choix du silex domine toujours pour le façonnage des outils, il s'équilibre avec la quartzite pour les déchets de débitage.

Au Solutréen récent, on assiste à un approvisionnement en silex de qualité locale (variété S2) ou distante (variétés S4 et S6). À la fin du Solutréen récent et au Badegoulien, les ressources locales (quartzites et, dans une moindre mesure, silex) deviennent prépondérantes. Au Magdalénien inférieur, l'approvisionnement en silex de qualité indifféremment locale (variété S1) ou distante (variétés S3 et S5) réapparaît. À l'Azilien enfin, l'abondance de la quartzite (variété QI) et la présence du silex de variété S1 traduit un approvisionnement en matières premières essentiellement locales. L'indétermination sur une origine précise des matières premières en silex ne permet toutefois pas d'en déduire des distances et des directions dans les déplacements pour l'approvisionnement en silex.

Des changements de stratégies d'approvisionnement en matières premières sont significatifs, et certainement liés aux variations climatiques. L'approvisionnement local à la fin du Solutréen récent et au Badegoulien traduit le maximum glaciaire. Le retour à l'approvisionnement en silex au Magdalénien inférieur traduit la circulation sur la côte cantabrique et sans doute vers le sud du bassin aquitain. Par contre, à l'Azilien, l'approvisionnement en silex et en quartzite locaux démontre un changement de comportement des chasseurs, limités à un territoire côtier, remontant plus profondément en altitude dans les montagnes cantabriques, et exploitant au maximum toutes les ressources locales disponibles.

\section{- Le débitage}

Les données disponibles publiées ne permettent pas de reconstituer les chaînes opératoires du silex couche par couche. Seules des études globales ont pu être entreprises (fig. 7) pour déceler les variations les plus significatives à travers la séquence stratigraphique, (Straus et Clark, 1986b; Straus et al., 1986).

Un rapport Débitage/Outillage maximum dans les couches 6 à 16 et dans les couches 25 à 28 nous conduit dans un premier temps à confirmer l'hypothèse de L.G. Straus, à savoir un débitage effectué sur le site même pour la fin du Solutréen récent (couches 6 et 7), le Badegoulien cantabrique (couches 8 à 16) et l'Azilien (couches 25 à 28), (Straus et al., 1986, p. 205). Au ProtoSolutréen (couches 1 et $2+3$ ), au début du Solutréen récent (couches 4 et 5) et au Magdalénien inférieur (couches 17 à 20), la réduction de ce rapport indique par contre l'importation de produits déjà partiellement élaborés : nucleus mis en forme, supports laminaires et lamellaires pré-débités... Au Magdalénien supérieur (?) (couches 21-23 et 24) et à l'Azilien (couches 25 à 28), on assiste à la réduction de la production des lamelles et à l'augmentation modérée des lames et des éclats.

Les variations globales mises en évidence sur la figure 7 permettent en outre de modéliser les types de débitage rencontrés dans la séquence de la Riera:

- Proto-Solutréen : débitage mixte : sur le site et par importation de produits semi-finis, orienté vers la production de lames et de lamelles;

- Solutréen récent : débitage mixte : sur le site et par importation de produits semi-finis, orienté vers la production de lames et de lamelles, puis, à partir de la couche 6 , substitution progressive par un débitage essentiellement sur le site, orienté vers la production d'éclats;

\begin{tabular}{|c|c|c|c|c|c|c|c|c|c|c|c|c|c|c|c|c|}
\hline & A & $\mathrm{B}$ & $\mathrm{C}$ & $\mathrm{D}$ & E & $\mathrm{F}$ & $\mathrm{G}$ & $\mathrm{H}$ & 0 & $\mathrm{Q}$ & $\mathrm{R}$ & $\mathrm{U}$ & $\mathrm{W}$ & $\mathrm{AA}$ & BB & Silex \\
\hline Minimum & 0,6 & 3,0 & 0,3 & 0,0 & 0,0 & 0,0 & 0,0 & 0,0 & 0,0 & 0,0 & 0,0 & 0,0 & 0,0 & 0,0 & 0,0 & 12,9 \\
\hline Maximum & 16,5 & 22,3 & 7,5 & 14,6 & 3,9 & 3,7 & 1,4 & 5,1 & 3,9 & 14,3 & 18,4 & 9,2 & 5,9 & 1,3 & 5,2 & 75,5 \\
\hline Moyenne & 5,1 & 9,4 & 3,2 & 4,4 & 0,5 & 0,5 & 0,4 & 0,8 & 0,6 & 4,1 & 1,5 & 1,0 & 2,0 & 0,2 & 0,2 & 34,4 \\
\hline Écart-type & 4,2 & 5,7 & 2,2 & 2,9 & 1,0 & 0,9 & 0,4 & 1,2 & 1,0 & 3,5 & 3,5 & 1,8 & 1,5 & 0,4 & 1,0 & 16,5 \\
\hline
\end{tabular}

\begin{tabular}{|l|c|r|r|r|r|r|r|c|}
\cline { 2 - 9 } \multicolumn{1}{c|}{} & I & \multicolumn{1}{c|}{ J } & \multicolumn{1}{c|}{ K } & \multicolumn{1}{c|}{ L } & \multicolumn{1}{c|}{ P } & \multicolumn{1}{c|}{ S } & \multicolumn{1}{c|}{ V } & Quartzite \\
\hline Minimum & 14,3 & 0,0 & 0,0 & 0,0 & 0,0 & 0,0 & 0,0 & 24,5 \\
\hline Maximum & 75,0 & 17,6 & 2,5 & 10,1 & 13,9 & 7,3 & 10,1 & 87,2 \\
\hline Moyenne & 47,0 & 8,4 & 0,3 & 2,4 & 1,5 & 0,7 & 0,6 & 65,5 \\
\hline Écart-type & 17,3 & 4,4 & 0,6 & 2,5 & 2,9 & 1,8 & 1,9 & 16,4 \\
\hline
\end{tabular}

\begin{tabular}{|r|r|c|c|c|c|}
\hline \multicolumn{1}{|c|}{$\mathrm{T}$} & \multicolumn{1}{c|}{$\mathrm{N}$} & $\mathrm{X}$ & $\mathrm{Y}$ & $\mathrm{Z}$ & Divers \\
\hline 0,0 & 0,0 & 0,0 & 0,0 & 0,0 & 0,0 \\
\hline 19,1 & 10,2 & 3,8 & 5,0 & 2,7 & 21,4 \\
\hline 2,6 & 0,8 & 1,2 & 0,5 & 0,5 & 5,5 \\
\hline 5,1 & 2,0 & 1,3 & 1,1 & 0,7 & 5,9 \\
\hline
\end{tabular}

Tab. 1 - Variabilité de la présence des matières premières dans la séquence archéologique de la Riera. 


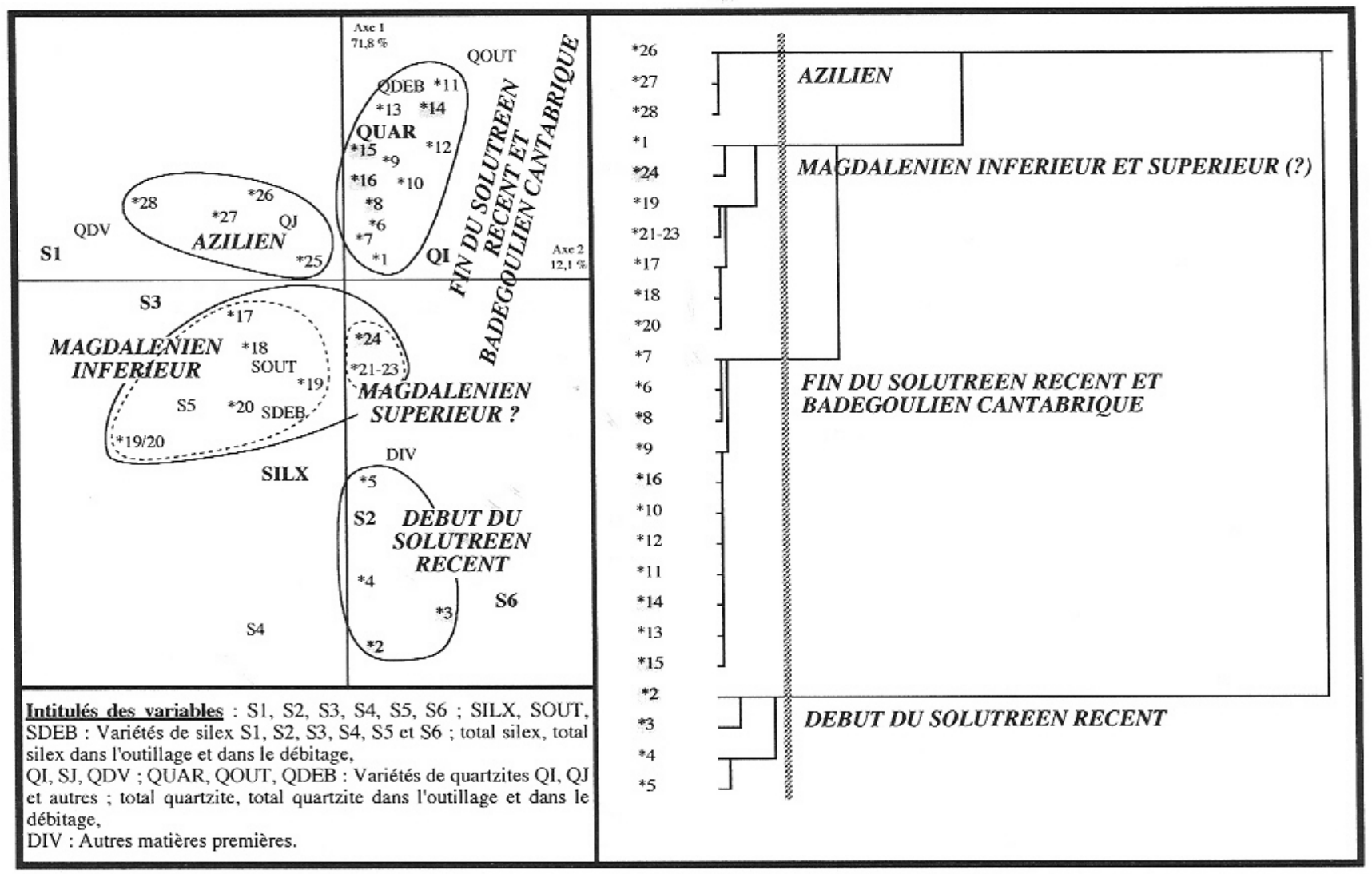

Fig. 6 - Approvisionnement en matières premières : plan factoriel 1-2 de l'analyse factorielle des correspondances et arbre de la classification automatique (les variables responsables de la signification des axes sont figurées en gras).

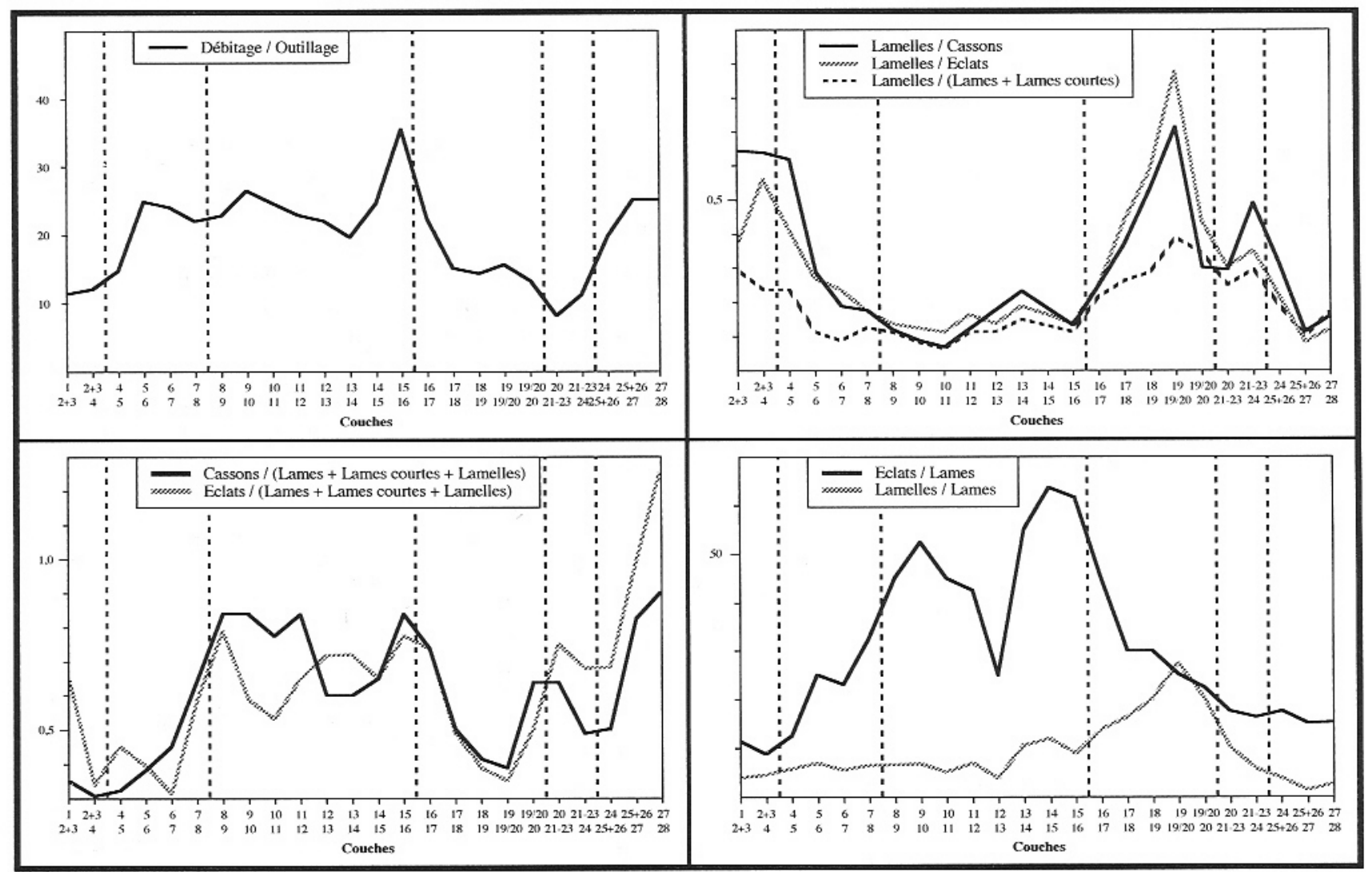

Fig. 7 - Le débitage dans la séquence de la Riera. 


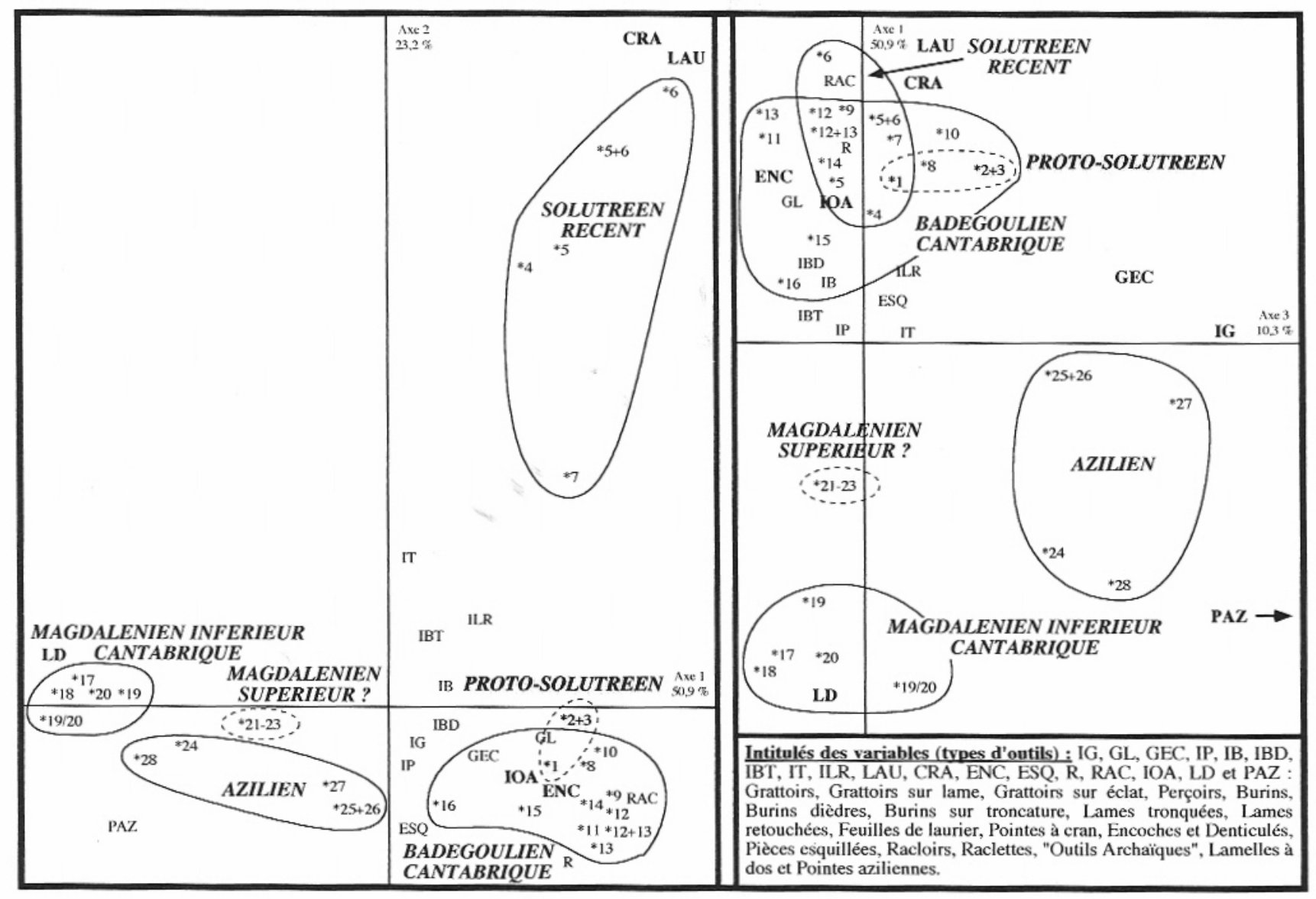

Fig. 8 - Structuration des industries lithiques de la séquence de la Riera : plans factoriels 1-2 et 1-3 de l'analyse factorielle des correspondances (les variables responsables de la signification des axes sont figurées en gras).

- Badegoulien : débitage sur le site, orienté essentiellement vers la production d'éclats;

- Magdalénien inférieur : débitage mixte : sur le site et par importation de produits semi-finis, orienté vers la production de lames et de lamelles;

- Magdalénien supérieur (?) et Azilien : débitage sur le site, orienté essentiellement vers la production de lames et d'éclats.

\section{Les outillages lithiques}

La figure 8 présente les résultats de l'analyse factorielle des correspondances des niveaux archéologiques de la séquence de la Riera, (Straus et Clark, 1986b). Le premier axe factoriel $(50,9 \%$ d'inertie) oppose des industries riches en encoches, en denticulés et/ou en outils “archaïques" à des industries riches en lamelles à dos. Le deuxième axe (23,2\% d'inertie) met en évidence l'originalité des niveaux 4 à 7 , caractérisés par le développement des feuilles de laurier et/ou des pointes à cran. Le troisième axe (10,3\% d'inertie) révèle la singularité des niveaux 24 à 28, caractérisés par une forte proportion de grattoirs, de grattoirs sur éclat et/ou de pointes aziliennes. Le quatrième axe enfin $(5,1 \%$ d'inertie) oppose des industries riches en grattoirs à des industries riches en pièces esquillées et/ou en pointes aziliennes. Une classification automatique, effectuée sur les quatre premières coordonnées factorielles, met en évidence une structure de partition en 4 faciès typologiques révélant six faciès chronologiques:

- couches 1 et 2 + 3: Proto-Solutréen, caractérisé par le développement des outils "archaïques", encoches et denticulés essentiellement ( $c$. infra);

- couches 4 à 7 : Solutréen récent, caractérisé par l'abondance des feuilles de laurier et des pointes à cran;

- couches 8 à 16 : Badegoulien cantabrique, caractérisés par le développement des outils "archaïques" : encoches et denticulés essentiellement;

- couches 17 à 20: Magdalénien inférieur, caractérisé par un grand développement des lamelles à dos ;

- couches 21 à 23 : Magdalénien (supérieur?), à plus rares lamelles à dos (pauvre);

- couches 24 à 28: Azilien, caractérisé par l'importance des lamelles à dos, le développement des grattoirs, dont de nombreux exemplaires courts, et des pointes aziliennes.

L'attribution au Proto-Solutréen des couches inférieures de la Riera a été déjà démontrée par une analyse des correspondances sur des niveaux gravettiens récents, ex-Aurignacien $\mathrm{V}$ et solutréens inférieurs de 
plusieurs stratigraphies de sites du sud-ouest de l'Europe sud-occidentale ${ }^{6}$, (déjà publiée dans Djindjian, 1996, fig. 1; Bosselin et Djindjian, 1997, fig. 2).

Une analyse typologique plus approfondie de ces faciès archéologiques sera développée ultérieurement.

\section{L'analyse \\ de l'industrie osseuse}

Devant la pauvreté de l'industrie osseuse, 144 outils et 138 fragments pour l'ensemble de la séquence, il convient de rester prudent quant aux conclusions pouvant déboucher d'une telle étude. Nous nous sommes donc efforcés de minimiser les variabilités liées à ce manque de représentativité. Pour cela, nous avons sélectionné, à partir de la typologie utilisée par M.R. Gonzales-Mirales (Gonzales-Mirales, 1986), 8 variables agrégées : les sagaies entières à base en biseau simple, les sagaies entières de section circulaire, ovalaire ou quadrangulaire et les dents perforées pour les outils entiers, les fragments de sagaie de section circulaire ou quadrangulaire et les fragments de fines aiguilles pour les débris. L'étude a été conduite sur des données “lissées", tout d'abord en agrégeant les couches 2 à 2 , puis en calculant, pour chaque ensemble, la fréquence de chaque variable, et enfin en éclatant ces variables en 4 modalités exclusives entre elles.

Ce tableau (niveaux archéologiques x modalités de variables) a été ensuite traité par analyse factorielle des correspondances (fig. 9A). Le premier axe factoriel (48,8\% d'inertie) oppose les sagaies (entières ou fracturées) de section quadrangulaire aux sagaies entières de section ovalaire. Le deuxième axe (20,4\% d'inertie) oppose les sagaies entières à base en biseau simple et les dents aux sagaies entières de section circulaire ${ }^{7}$. Le troisième axe enfin (13,5\% d'inertie) met en évidence l'originalité des niveaux à fragments de pointes fines (aiguilles).

Une classification automatique (fig. 9B), effectuée sur les coordonnées des axes 1 et 3 , met en évidence une structure de partition en 3 classes:

- c. $(2+3) / 4$ à 16/17 : Solutréen récent et Badegoulien cantabrique, caractérisés par la fréquence des sagaies entières, à base en biseau simple, de section circulaire ou ovalaire; des fragments de sagaies, de section circulaire ; des fines aiguilles; et des dents perforées ; - c.17/18 à 21-23/24: Magdalénien (inférieur et supérieur [?]), caractérisé par la fréquence des sagaies

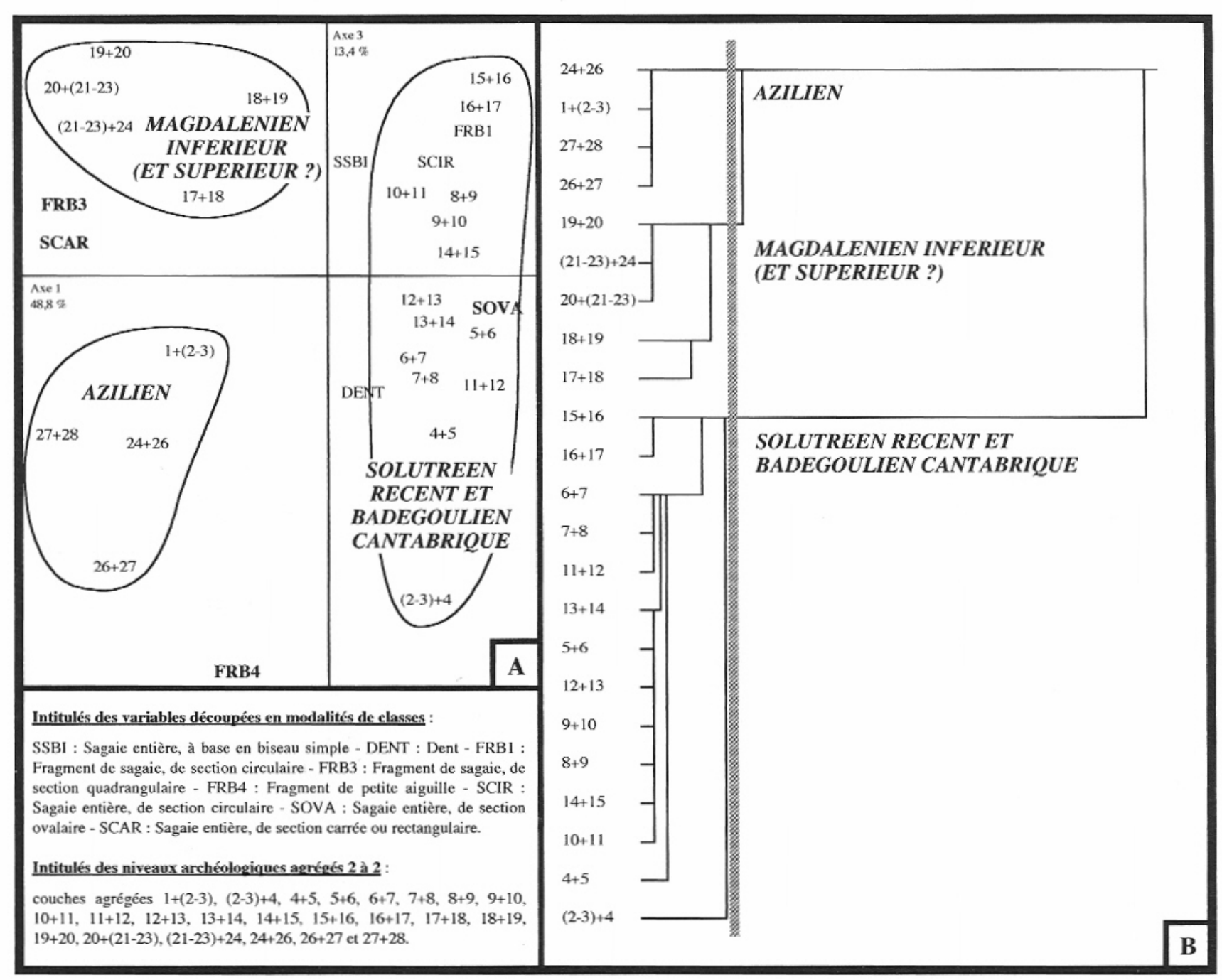

Fig. 9 - Structuration des industries osseuses de la séquence de la Riera. 9A : plan factoriel 1-2 de l'analyse factorielle des correspondances (les variables responsables de la signification des axes sont figurées en gras); $9 \mathbf{B}$ : arbre de la classification automatique. 


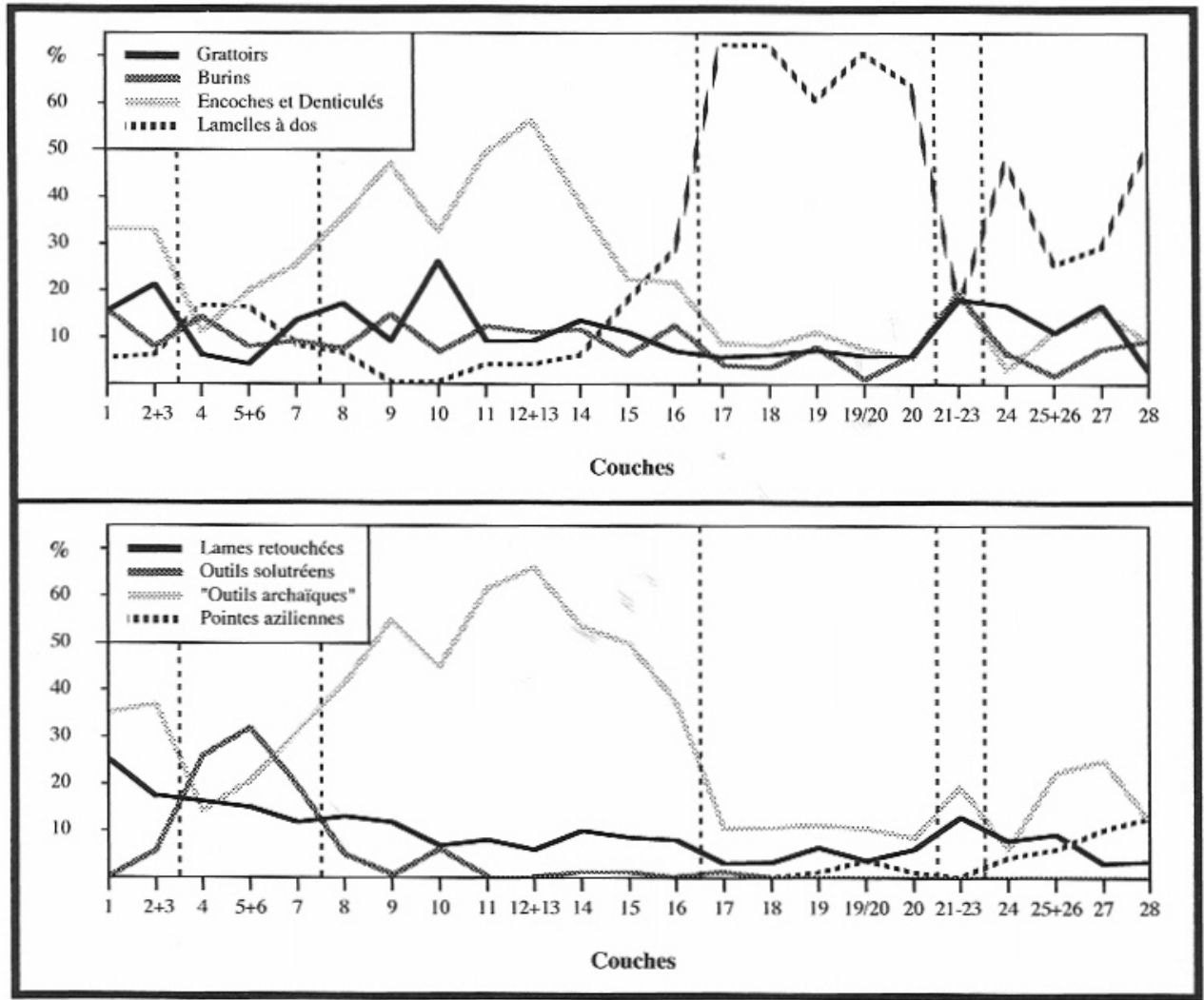

Fig. 10 - Variations des principaux groupes typologiques dans la séquence de la Riera. entières à base en biseau simple et des fragments de sagaies, de section quadrangulaire ;

- c.24/26 à 27/28: Azilien, caractérisé par la fréquence des sagaies entières ou fragmentées, de section quadrangulaire; des fines aiguilles; et des harpons plats aziliens.

Les conclusions mettent en évidence l'homogénéité de l'industrie osseuse pour l'ensemble Solutréen/Badegoulien d'une part (sagaies à base en biseau simple de section circulaire ou ovalaire) et l'apparition caractéristique des sagaies de section quadrangulaire dès le Magdalénien inférieur cantabrique, (Utrilla-Miranda, 1981).

\section{Les faciès lithiques}

\section{Continuités et discontinuités typologiques}

La figure 10 présentent les variations des principaux groupes typologiques (grattoirs, burins, lames retouchées, outils solutréens, outils "archaïques", encoches et denticulés, lamelles à dos et pointes aziliennes) dans la séquence archéologique de la Riera.

Les grattoirs et les burins, dont les pourcentages fluctuent de façon aléatoire entre 1,3 et $26,8 \%$, ne présentent pas de variations en rapport avec une quelconque évolution en stratigraphie. Toutefois comme prévu, les grattoirs courts sont plus abondants dans l'Azilien (couches 24 à 28).

Les lames retouchées décroissent régulièrement de bas en haut dans la séquence.

Les outils solutréens apparaissent dans la couche $2+3$, puis se développent nettement dans les niveaux 4 à 7 , avant de perdurer de façon sporadique dans l'ensemble 8-17. Ils mettent donc en évidence l'existence de deux discontinuités typologiques: d'une part entre les couches $2+3$ et 4 , d'autre part entre les couches 7 et 8 . S'agit-il d'une pollution stratigraphique ou d'une continuité dans les niveaux badegouliens, phénomène déjà signalé par ailleurs, (Bordes, 1958; Trotignon, 1984)?

Corrélativement, les outils "archaïques" montrent une évolution inverse. Abondants à la base (couches 1 et $2+3$ ), ils atteignent, après une décroissance significative dans les niveaux 4 à 7 , leur maximum dans les couches 8 à 16. Une nouvelle diminution survient ensuite à partir de la couche 17. Ce groupe est essentiellement constitué d'encoches et de denticulés, mis à part dans les couches 15 et 16 où l'on assiste au développement des pièces esquillées, des racloirs et des raclettes. Cela confirme donc la double cassure typologique existant entre les couches 7 et 8 d'une part, 16 et 17 d'autre part.

Les lamelles à dos viennent renforcer l'existence de ces discontinuités puisqu'on ne les rencontre en proportion significative que dans les couches 17 à 28 .

Enfin, les pointes aziliennes n'apparaissent qu'au sommet de la séquence, dans les couches 24 à 28 .

\section{Esquisse typologique des faciès lithiques}

En conséquence, la structuration des industries lithiques de la séquence de la Riera s'effectue essentiellement à partir des variations des groupes typologiques des grattoirs courts, des lames retouchées, des outils solutréens, des outils "archaïques", des encoches et denticulés, des lamelles à dos et des pointes aziliennes. 
Un profil typologique des faciès lithiques est présenté sur la figure 11, à partir des valeurs moyennes calculées pour chacune des classes définies par la classification automatique :

- Proto-Solutréen (couches 1 et $2+3$ ) : abondance des outils " archaïques", essentiellement des encoches et denticulés, supériorité des grattoirs sur les burins, proportion importante de grattoirs courts, fréquence élevée des lames retouchées et rareté des lamelles à dos;

- Solutréen récent (couches 4 à 7) : supériorité modérée des burins sur les grattoirs, dont les exemplaires courts diminuent, fréquence moyenne des lames retouchées et des lamelles à dos, développement des outils solutréens et forte diminution des outils "archaïques", essentiellement constitués d'encoches et denticulés ;

- Badegoulien cantabrique (couches 8 à 16) : rapport IG/IB généralement supérieur à l'unité, quasi disparition des outils solutréens et des lamelles à dos et développement massif des outils "archaïques", essentiellement constitués d'encoches et denticulés.

La variabilité de ce faciès est assurée par les fluctuations dans le pourcentage et la composition des outils “archaïques", et par la fréquence des lamelles à dos. Plusieurs niveaux se distinguent de l'ensemble: la couche 8 , caractérisée par une plus faible proportion d'encoches et denticulés, le développement des grattoirs, des outils solutréens et des lamelles à dos; les couches 15 et 16 , caractérisées par la substitution partielle des encoches et denticulés par les pièces esquillées, les racloirs et les raclettes et l'abondance croissante des lamelles à dos. La mise en évidence de perturbations et de remaniements dans les dépôts contemporains des couches 8 et $15-16$ permet de conclure à des mélanges d'industries plutôt qu'à l'existence des transitions Solutréen/Badegoulien et Badegoulien/Magdalénien inférieur à la Riera ;

- Magdalénien inférieur cantabrique (couches 17 à 20) : l'abondance des lamelles à dos écrase les autres catégories de l'outillage (grattoirs, burins, outils " archaïques").

L'érosion du sommet de la séquence du Magdalénien inférieur a très certainement entraîné des mélanges d'industries du Magdalénien inférieur et du Magdalénien supérieur (couche 20);

- Magdalénien supérieur [?] (couches 21 à 23 et 24) : les couches 21 à 23 et 24 , dont l'échantillon est toutefois statistiquement limité pour la couche 21 à 23 , se caractérisent par une fréquence moins élevée en lamelles à dos;

- Azilien (couches 25 à 28) : faible décroissance des lamelles à dos et développement des grattoirs courts, des pointes aziliennes et, dans une moindre mesure, des outils "archaïques" (encoches et denticulés).

\section{Conclusion}

En conclusion, cette étude nous a permis de distinguer, dans la séquence "solutréenne" définie par L.G. Straus (couches 2 à 18), deux entités culturelles autonomes (Straus, 1986a et c; Straus et Clark, 1986c) : le Solutréen récent (couches 4 à 7) et une industrie d'aspect régressif, caractérisée par le développement des outils

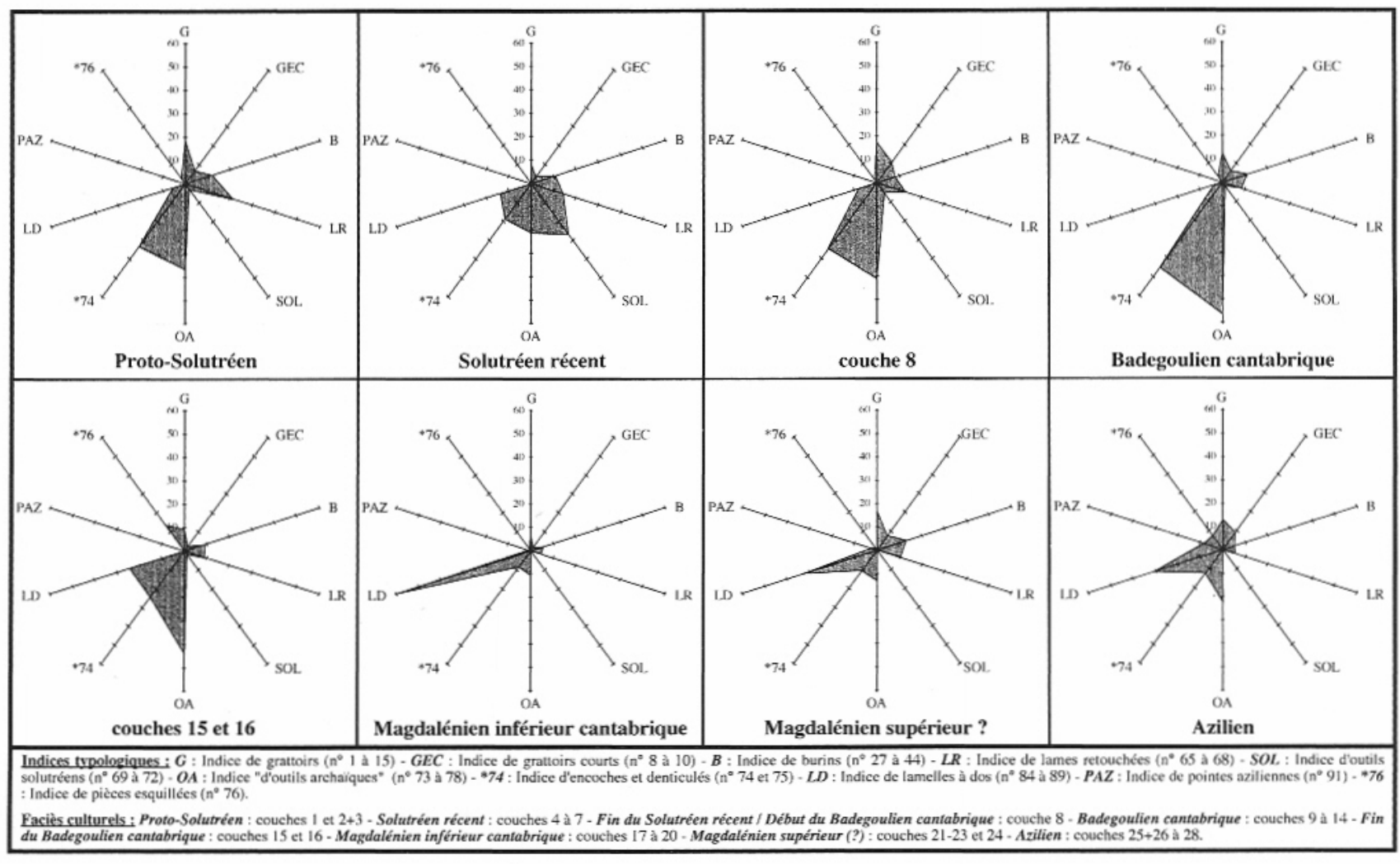

Fig. 11 - Esquisse typologique des faciès industriels lithiques de la séquence de la Riera (les pourcentages figurés correspondent aux valeurs moyennes calculées sur l'ensemble des niveaux de chaque faciès). 
"archaïques", essentiellement des encoches et denticulés, l'extrême rareté des outils solutréens, des lamelles à dos et un rapport IG/IB variable, mais généralement supérieur à l'unité, et que nous proposons de nommer désormais Badegoulien cantabrique (couches 8 à 16), la couche $2+3$ étant proto-solutréenne, les couches 17 et 18 magdaléniennes.

Le Badegoulien cantabrique se différencie des ensembles antérieur (Solutréen) et postérieur (Magdalénien inférieur) tant par une composition typologique originale (abondance des outils "archaïques", rareté des outils solutréens et des lamelles à dos) que par des stratégies d'approvisionnement essentiellement locales et peu diversifiées en matières premières $(73 \%$ de quartzites des rives du Rio Bedón en moyenne) et en ressources alimentaires (cerf, bouquetin et chamois du biotope local) et par un débitage intégralement réalisé sur le site, conséquences d'un isolement local des populations de chasseurs-cueilleurs lors du maximum glaciaire, situation qui se rencontre toutefois dès la fin du Solutréen récent.
La révision du cadre paléoclimatique met en évidence l'omniprésence d'un contexte froid pour la base de la séquence (couches 1 à 21), traduit par une végétation ouverte variant suivant le taux d'humidité (steppe ou lande). Seule la partie sommitale de la stratigraphie voit le développement des Juniperus dans un premier temps (couches 22 et 23), puis d'une boulaie à Filicales (couches 24 à 28), signes d'une nette amélioration thermique correspondant aux dernières manifestations du tardiglaciaire (oscillation tardiglaciaire Bølling/Allerød), puis le Pré-Boréal et le Boréal. De nombreuses lacunes de sédimentation ou des vidanges sont perceptibles ${ }^{8}$. Corrélativement, les industries préhistoriques associées montrent des indices de remaniements significatifs, ce qui empêche de conclure à l'existence de phases de transition entre Solutréen et Badegoulien cantabrique (couche 8 ) et entre Badegoulien cantabrique et Magdalénien inférieur (couches 15 et 16). Un tableau résumant l'occupation préhistorique du site de la Riera dans son cadre paléoclimatique est proposé sur la figure 12 .

\begin{tabular}{|c|c|c|c|c|c|}
\hline CHRONOLOGIE & PHASES CLIMATIQUES & CLIMAT & DATATIONS 14C & INDUSTRIES & COUCHES \\
\hline $9000 \mathrm{BP}$ & BOREAL & $\begin{array}{l}\text { Tempéré, } \\
\text { Très Humide }\end{array}$ & $(8650 \pm 300 \mathrm{BP})$ & ASTURIEN & $\begin{array}{l}30 \\
29\end{array}$ \\
\hline \multirow[b]{2}{*}{$11000 \mathrm{BP}$} & Lacune : Dryas III & - & - & & - \\
\hline & ALLERØD & Tempéré, Humide & $(10630 \pm 120 \mathrm{BP})$ & AZILIEN & 28 à 25 \\
\hline $12000 \mathrm{BP}$ & - & - & - & Remaniement & 24 \\
\hline $13000 \mathrm{BP}$ & $\begin{array}{l}\text { BØLLING } \\
\text { FIN DRYAS I }\end{array}$ & $\begin{array}{l}\text { Moins Froid, } \\
\text { Plus Humide }\end{array}$ & $\begin{array}{l}12630 \pm 300 \mathrm{BP} \\
12360 \pm 670 \mathrm{BP}\end{array}$ & $\begin{array}{l}\text { MAGDALENIEN } \\
\text { SUPERIEUR? }\end{array}$ & 23 à 20 sup. \\
\hline \multicolumn{6}{|l|}{$14000 \mathrm{BP}$} \\
\hline \multicolumn{6}{|l|}{$15000 \mathrm{BP}$} \\
\hline $16000 \mathrm{BP}$ & DRYAS I & Froid, Sec & $\begin{array}{l}15520 \pm 350 \mathrm{BP} \\
16420 \pm 430 \mathrm{BP} \\
16900 \pm 200 \mathrm{BP} \\
17070 \pm 230 \mathrm{BP}\end{array}$ & $\begin{array}{l}\text { MAGDALENIEN } \\
\text { INFERIEUR } \\
\text { CANTABRIQUE }\end{array}$ & 20 inf. à 17 \\
\hline $17000 \mathrm{BP}$ & Fin Lascaux / Début Dryas & - & $17225 \pm 350 \mathrm{BP}$ & Remaniement & 16 et 15 \\
\hline \multirow{2}{*}{$18000 \mathrm{BP}$} & $\begin{array}{c}\text { "EPISODE } \\
\text { DE LASCAUX" }\end{array}$ & Froid, Humide & $(17210 \pm 350 \mathrm{BP})$ & $\begin{array}{l}\text { BADEGOULIEN } \\
\text { CANTABRIQUE }\end{array}$ & 14 à 9 \\
\hline & $\begin{array}{c}\text { Lacune : inter } \\
\text { "Laugerie" / "Lascaux" }\end{array}$ & - & - & Remaniement & 8 \\
\hline $19000 \mathrm{BP}$ & $\begin{array}{l}\text { "EPISODE DE } \\
\text { LAUGERIE" }\end{array}$ & Froid, Humide & - & $\begin{array}{l}\text { SOLUTREEN } \\
\text { RECENT }\end{array}$ & - 7 à 4 \\
\hline $000 \mathrm{BP}$ & Lacune & - & - & - & - \\
\hline \multicolumn{6}{|l|}{$21000 \mathrm{BP}$} \\
\hline $22000 \mathrm{BP}$ & $\begin{array}{l}\text { PLENIGLACIAIRE } \\
\text { DU WURM RECENT }\end{array}$ & Froid, Sec & $\begin{array}{l}(20360 \pm 450 \mathrm{BP}) \\
(20860 \pm 410 \mathrm{BP})\end{array}$ & $\begin{array}{c}\text { PROTO- } \\
\text { SOLUTREEN }\end{array}$ & 3 à 1 \\
\hline
\end{tabular}

Fig. 12 - Synthèse chronostratigraphique et paléoclimatique de la séquence industrielle de la Riera. 


\section{L'ESPAGNE CANTABRIQUE ENTRE 22000 ET 16000 B.P.}

\section{Les données}

En Espagne cantabrique, les sites de référence récemment fouillés pour la période 22000 B.P.-16000 B.P. sont, outre la Riera, Las Caldas (Corchon-Rodriguez, 1981), Cueva Morin (Gonzales-Echegaray et Freeman, 1971, 1973), Cueva Chufin (Cabrera-Valdes, 1977), Amalda (Altuna et al., 1990), Ekain (Altuna et Merino, 1984), Rascaño (Gonzales-Echegaray et BarandiaranMaetzu, 1981), Erralla (Altuna et al., 1985), el Juyo (Barandiaran-Maetzu et al., 1985) et Abauntz, (UtrillaMiranda, 1982).

Des recherches sont en cours (Barandiaran-Maetzu, 1996 ; Bernaldo de Quiros, 1996; Fortea-Perez, 1996 ; Rasilla-Vives, 1990b) au Cueto de la Mina (Hoyos-Gomez et Rasilla-Vives, 1994 ; Rasilla-Vives, 1990a), la Viña (Fortea-Perez, 1990, 1992, 1995), la Lluera (Rodriguez-Asensio, 1990, 1992), Llonin (Fortea-Perez et al., 1992, 1995), Las Caldas (Corchon-Rodriguez, 1990, 1992, 1995), Abauntz (fouilles P. Utrilla-Miranda) et au Castillo (fouilles V. Cabrera-Valdes).

Des données complémentaires proviennent de travaux plus anciens, mais dont le contrôle stratigraphique est satisfaisant, à Cueva Oscura, Cova Rosa, el Cierro, Cueto de la Mina, Altamira, Hornos de la Peña, Castillo, Pasiega, Aitzbitarte IV, Santimamine, Ermittia, Urtiaga, Lumentxa, Lloseta, Paloma et Balmori, (Bernaldo de Quiros, 1982 ; Cabrera-Valdes, 1984 ; Moure-Romanillo et al. éd., 1995 ; Rasilla-Vives éd., 1994 ; Straus, 1983 ; Utrilla-Miranda, 1981).

\section{L'analyse des industries lithiques}

La figure 13 présente les résultats de l'analyse factorielle des correspondances des niveaux archéologiques solutréens et magdaléniens situés sur la plage 22000 16000 B.P. dans les Cantabres. Le premier axe factoriel (46,8\% d'inertie) oppose les niveaux à nombreuses lamelles à dos à ceux à nombreux outils "archaïques" (encoches, denticulés, pièces esquillées, racloirs et raclettes). Le deuxième axe (17,4\% d'inertie) oppose les niveaux à nombreux grattoirs et/ou burins à ceux à nombreux outils " archaïques" et/ou feuilles de laurier. Le troisième axe enfin ( $16,6 \%$ d'inertie) oppose les niveaux à nombreux outils solutréens (feuilles de laurier et pointes à cran) à ceux à nombreux encoches et denticulés. Une classification automatique, effectuée sur ces trois premières coordonnées factorielles, met en évidence une structure de partition en quatre faciès lithiques nommés ici, (Bosselin et Djindjian, 1988) :

- Solutréen récent : caractérisé par le développement des outils solutréens (feuilles de laurier et pointes à cran), la fréquence moyenne des outils " archaïques", des grattoirs et des burins et la présence significative des lamelles à dos;

- Badegoulien cantabrique : caractérisé essentiellement par le foisonnement des outils " archaïques", composés principalement d'encoches et de denticulés, la rareté des lamelles à dos et la présence sporadique d'outils solutréens ;

- Magdalénien inférieur cantabrique de faciès M0/M1: caractérisé par l'abondance des grattoirs et des burins, un rapport IB/IG généralement supérieur à l'unité, la supériorité des burins dièdres sur les exemplaires sur

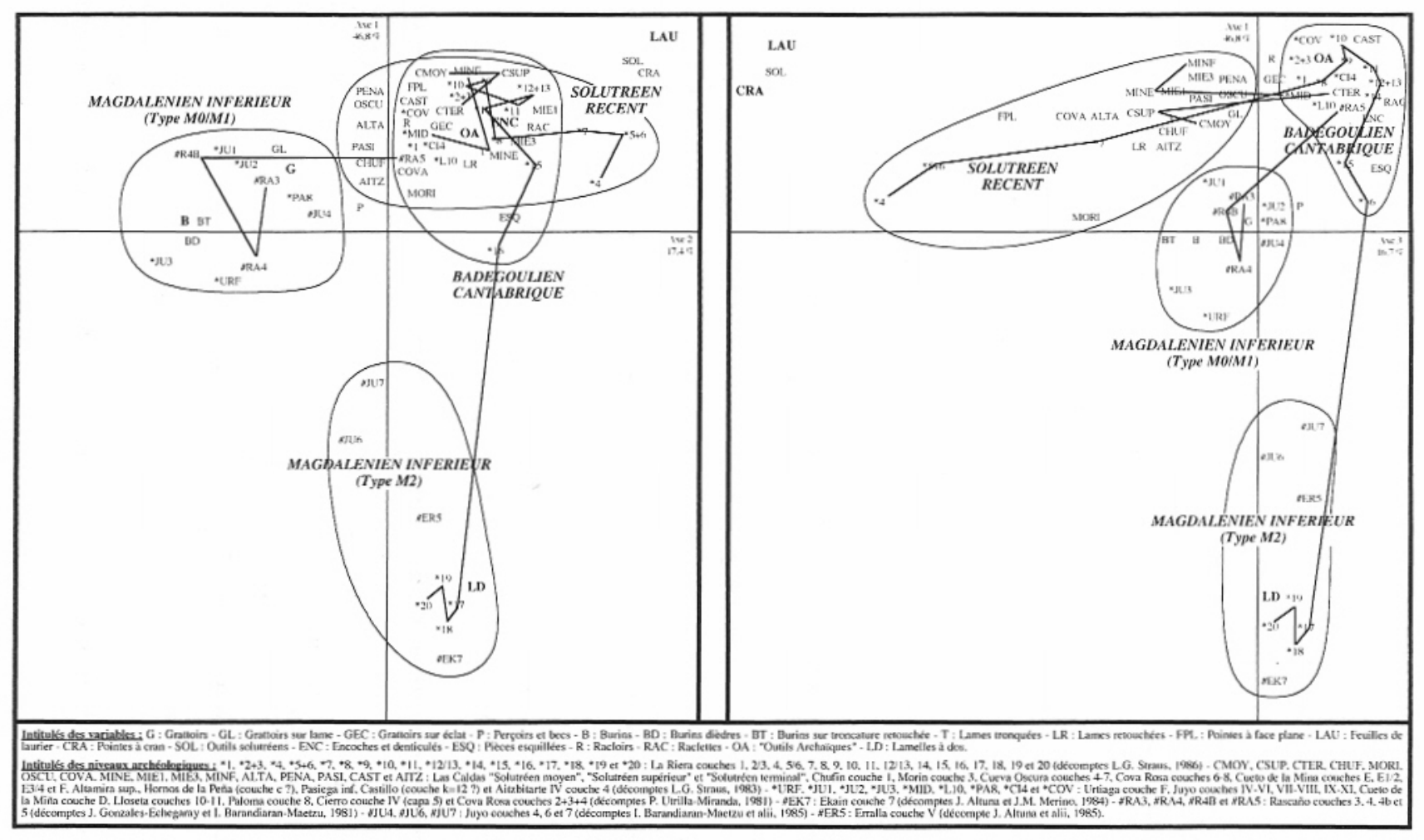

Fig. 13 - Structuration des industries lithiques en Espagne atlantique entre 22000 B.P. et 16000 B.P. : plans factoriels 1-2 et 1-3 de 1'analyse factorielle des correspondances (les variables responsables de la signification des axes sont figurées en gras). 
troncature retouchée, la rareté des outils "archaïques" et un pourcentage modéré de lamelles à dos ; - Magdalénien inférieur cantabrique de faciès M2 : caractérisé par le foisonnement des lamelles à dos et, corrélativement, la faible représentation des autres catégories de l'outillage (grattoirs, burins, outils " archaïques”). Là encore, on constate des rapports IB/IG et $\mathrm{IBd} / \mathrm{IBt}$ généralement supérieurs à l'unité.

En conséquence, la structuration des industries lithiques situées entre environ 22000 B.P. et 16000 B.P. en Espagne atlantique s'effectue, comme en Aquitaine (Bosselin et Djindjian, 1988), essentiellement à partir des variations des groupes typologiques des grattoirs, des burins, des lames retouchées, des outils solutréens, des outils " archaïques" et des lamelles à dos. Cette esquisse typologique des faciès lithiques permet de confirmer définitivement l'existence du Badegoulien cantabrique, en tant qu'entité à la fois distincte du Solutréen sous-jacent et du Magdalénien sus-jacent.

\section{L'analyse de l'industrie osseuse}

Malgré la pauvreté de l'industrie osseuse recueillie lors des fouilles modernes et l'incertitude quant à la position stratigraphique précise de certains outils "caractéristiques” issus des recherches anciennes, parfois considérés comme "fossiles directeurs" dans les constructions antérieures, son étude permet d'affiner la structure mise en évidence à partir des seuls outillages lithiques, (Adan-Alvarez, 1994 ; Corchon-Rodriguez, 1994 ; Straus, 1983 ; Utrilla-Miranda, 1981, 1986) :

- au Solutréen récent, l'industrie osseuse est dominée par les sagaies à biseau simple, de section circulaire ou ovalaire. Dans les ensembles récemment fouillés (Las Caldas, Chufin et la Riera), elles sont accompagnées de quelques bipointes de section circulaire, à méplat central, et de biseaux en lancette ("lengüeta") ou décorés en épi qui annoncent déjà le Badegoulien cantabrique sus-jacent;

- au Badegoulien, l'industrie osseuse conserve globalement les mêmes caractéristiques : domination des sagaies à biseau simple, de section circulaire ou ovalaire, et présence de bipointes de section circulaire, à méplat central. On assiste en outre au développement d'outils diagnostiques du Badegoulien français: biseaux striés en épi ou en chevrons, biseaux en lancette et sagaies à fût décoré par motifs tectiformes ("technique de pseudo-excision"), (Utrilla-Miranda, 1986) ;

- au Magdalénien inférieur cantabrique, on assiste à l'apparition des sagaies de section quadrangulaire, avec ou sans rainure. Les sagaies de section circulaire ou ovalaire sont toujours présentes, quoique moins nombreuses.

\section{Le contexte paléoclimatique et les datations absolues}

La reconstitution du cadre paléoclimatique de la séquence cantabrique se heurte à de nombreuses difficultés: l'ancienneté de la plupart des recherches; la proximité de l'océan et ses conséquences sur les signaux climatiques; et l'existence de lacunes (vidanges) dans les remplissages, qui ont entraîné parfois des mélanges, compliquant l'interprétation des analyses sédimentologique et palynologique.

Le Solutréen récent se situe dans une phase froide et humide correspondant à "l'épisode de Laugerie" au Cueto de la Mina (couches VIa-VIb $=$ F ?), la Riera (couches 4 à 7), Las Caldas (couches 10 à 17), Cova Rosa (couches F1-F4 = 6 à 8 ?), Chufin (couche 1), Cueva Morin (couche 3), la Viña (couches VI et VIa), la Lluera (couche IX), Castillo et Hornos de la Peña, (BoyerKlein, 1980 ; Butzer, 1981 ; Hoyos-Gomez, 1981, 1995 ; Laville, 1986; Arl. Leroi-Gourhan, 1971, 1986). Pourrait-il perdurer dans le Dryas I inter Laugerie/Lascaux froid et sec à la Viña (couche V), Cueto de la Mina (couches $\mathrm{Va}-\mathrm{Vb}=\mathrm{E}$ ?), la Lluera (couches VI à VIII) et Cova Rosa (couches E3 à DE) ? mais l'absence de publication des industries et/ou l'ancienneté des fouilles ne permet pas de leur assigner une attribution culturelle (Solutréen ou Badegoulien) ou chronologique ("Laugerie" ou Dryas I) définitive, (Hoyos-Gomez, 1995).

À Las Caldas, le retour du froid serait perceptible dès la couche 9 ("Solutréen supérieur") puis s'amplifierait jusqu'à la couche 4 (Badegoulien), mais la présence d'une phase de ravinement plus humide entre les couches 6 et 7 (Corchon-Rodriguez, 1981; HoyosGomez, 1981, 1995 ; Paquereau dans Hoyos-Gomez, 1981) et l'érosion partielle des niveaux 7 à 9 nous amène plutôt à supposer qu'il pourrait s'agir des dernières manifestations de "l'épisode de Laugerie", rejetant alors la fin du Solutréen récent (couche 7) dans "l'épisode de Laugerie" et le début du Badegoulien (couche 6) dans le Dryas I.

Le Badegoulien est situé dans une phase froide et humide correspondant à "l'épisode de Lascaux" à la Riera (couches 9 à 16), Las Caldas (couches 3 à 6), Rascaño (couche 5) et peut-être au Cueto de la Mina (couche IVb = D ?), (Boyer-Klein, 1980, 1981; Corchon-Rodriguez, 1981 ; Hoyos-Gomez, 1981, 1995 ; Laville, 1986; Laville et Hoyos-Gomez, 1981; Arl. Leroi-Gourhan, 1986).

Le Magdalénien inférieur cantabrique enfin se situe dans une phase froide et sèche correspondant au Dryas I (phases Würm IV-Cantabres III à V) à Rascaño (couches 3 et 4), Erralla (couche V), la Paloma (couches 6 et 8), el Juyo (couches 4 à 13), Cueto de la Mina (couches III et $\mathrm{IVa}=\mathrm{C}-\mathrm{D}$ ?), Ekain (couche VII), la Riera (couches 17 à 20) et peut-être Las Caldas (couches 1 et 2-Sala II: couches VI à XIII) et Cova Rosa (couche $\mathrm{C}=2$ à 4 ?), (Areso, 1984; Boyer-Klein, 1981, 1985; Boyer-Klein et Arl. Leroi-Gourhan, 1985 ; Dupré, 1984 ; Hoyos-Gomez et Fumanal, 1985; Hoyos-Gomez et al., 1980 ; Laville, 1986 ; Arl. Leroi-Gourhan, 1986; Lopez, 1982).

Les données du paléoenvironnement soulignent l'omniprésence d'un contexte froid pour la séquence située entre 22000 B.P. et 16000 B.P. en Espagne atlantique. Seules des variations d'humidité, marquées par l'alternance Éricacées/Composées/Graminées dans la flore herbacée, sont perceptibles, et correspondent toujours à une dynamique de reforestation très limitée du 
paysage, en particulier pour les "épisodes" de Laugerie et de Lascaux. De nombreuses lacunes ont induit des remaniements ponctuels du matériel archéologique, ne permettant pas d'établir formellement l'existence des transitions Solutréen/Badegoulien et Badegoulien/ Magdalénien. L'absence apparente du Dryas I inter Laugerie/Lascaux en stratigraphie, pour les mêmes raisons, empêche de reconnaître une structure typologique à l'intérieur du Badegoulien cantabrique, au contraire de l'Aquitaine où deux phases chronologiques ont été clairement mises en évidence, (Bosselin et Djindjian, 1988).

Malgré leur forte variabilité, les datations absolues permettent de proposer des plages de dates compatibles avec ce cadre paléoclimatique. Ainsi, le Solutréen récent se situe entre environ 20500 B.P. et 19000 B.P., le Badegoulien entre environ 18500 B.P. et 17000 B.P. et le Magdalénien à partir de 17000 B.P. La publication des recherches actuelles permettra vraisemblablement de compléter et d'affiner cette première synthèse.

En conclusion, la prise en compte de l'ensemble des données disponibles en Espagne atlantique sur la période 22000 B.P.-16000 B.P., même si beaucoup d'entre elles proviennent de recherches anciennes dont la valeur peut paraître moindre, permet de confirmer l'existence du Badegoulien cantabrique, et sa nette différenciation du Solutréen récent et du Magdalénien inférieur. Du point de vue climatique, le Badegoulien cantabrique se développe principalement au cours de "l'épisode de Lascaux". Si l'on observe fréquemment les superpositions stratigraphiques Solutréen récent/ Badegoulien, Badegoulien/Magdalénien inférieur et Solutréen récent/Badegoulien/Magdalénien inférieur, les transitions entre ces différents faciès lithiques restent à établir. En particulier, le Badegoulien cantabrique n'apparaît pas, dans l'état actuel des connaissances, comme un "Solutréen en processus de désolutréanisation" (Rasilla-Vives, 1989, 1994a et b), un "Solutréen final" ou "terminal" (Corchon-Rodriguez, 1981, 1994), un "Solutréen désolutréanisé" (Straus, 1975) ou un "Magdalénien inférieur archaïque " (Utrilla-Miranda, 1981, 1989), mais du moins tout cela à la fois.

\section{LE BADEGOULIEN DU PORTUGAL}

Au Portugal, en Estramadoure, plusieurs sites " magdaléniens anciens" peuvent être en fait attribués à un Badegoulien portugais. Les industries sont caractérisées (Bicho, 1997 ; Marks et Mishoe, 1997) par l'abondance des outils en quartz et quartzite $(11 \%)$, un débitage faiblement laminaire $(7,5 \%)$, l'existence d'un substrat aurignacien et très peu de lamelles à dos, ce qui les rapproche du Badegoulien cantabrique comme du Magdalénien ancien méditerranéen, (Aura-Tortosa, 1995 ; Zilhao, 1997). Les neuf dates ${ }^{14} \mathrm{C}$ de Cabeço de Porto Marinho, comprises entre 17500 B.P. et 14000 B.P., semblent bien trop récentes pour la chronologie de cette industrie, attendue plus vieille que 17000 B.P., (Marks et Mishoe, 1997).

\section{CONCLUSION}

La révision de la séquence archéologique de la Riera nous a permis, outre de proposer une nouvelle interprétation de l'occupation chrono-climatique et culturelle de ce site, de mettre en évidence l'existence d'un faciès lithique encore jamais reconnu formellement en Espagne atlantique : le Badegoulien cantabrique. Par ses caractéristiques typologiques originales, abondance des grattoirs et des encoches-denticulés, rareté ou absence des outils solutréens et des lamelles à dos, il se différencie nettement du Solutréen récent sous-jacent et du Magdalénien inférieur sus-jacent.

Si les industries lithiques opposent clairement le Solutréen du Badegoulien, l'approvisionnement en matières premières, le débitage (pour la fin du Solutréen récent essentiellement) et l'outillage osseux laissent apparaître quelques convergences entre ces deux faciès, conséquences d'un cloisonnement des populations de chasseurs-cueilleurs lors du maximum glaciaire. Ce cloisonnement se retrouve d'ailleurs à l'échelle de l'Europe sud-occidentale où des faciès sensiblement contemporains, possédant un substrat typologique commun, développent des spécificités régionales ou géographiques ${ }^{10}$. Une analyse globale de ces entités nous permettra vraisemblablement de proposer, dans l'avenir, un nouveau modèle de peuplement (ou de déplacement de population) au cours du maximum glaciaire et de mieux connaître les rapports entre Solutréen, Badegoulien et Magdalénien en Aquitaine, dans les Cantabres et en Espagne levantine.

La paléoclimatologie place le Badegoulien dans le Dryas I inter Laugerie/Lascaux et surtout au cours de "l'épisode de Lascaux", dans un contexte toujours froid mais montrant des variations d'humidité marquées. Malheureusement, des hiatus d'occupation, liés à l'histoire géologique des abris dans cette région, nous empêchent de posséder des séquences complètes, où les niveaux archéologiques sont exempts de toute contamination par les industries antérieures ou postérieures. Cela ne nous permet pas de régler la question des transitions Solutréen/Badegoulien et Badegoulien/ Magdalénien inférieur ou de reconnaître une éventuelle structuration interne du Badegoulien cantabrique.

Remerciements : nous tenons à remercier très chaleureusement les auteurs qui nous ont aimablement fourni de précieux renseignements pour cette étude: en premier lieu L.G. Straus, pour l'envoi de la publication du site de la Riera, K. Mariezkurrena pour Erralla, M. de la Rasilla-Vives pour le Solutréen cantabrique, P. Utrilla-Miranda pour le Magdalénien inférieur " archaïque" cantabrique, Ch. Leroyer et B. Kervazo pour leur indications concernant l'interprétation des analyses palynologiques et sédimentologiques.

Les programmes d'analyse factorielle des correspondances (ANCORR), de classification ascendante hiérarchique (CAH2CO) et d'aide à l'interprétation d'une analyse des correspondances (AIDAFC) sont extraits de la bibliothèque ADDAD 91, maintenant disponible sur système de micro-informatique Apple Macintosh ${ }^{\mathrm{R}}$. Nous remercions ici Monsieur Jean-Pierre Fénelon de nous avoir fourni ce logiciel. 


\section{NOTES}

(1) Il est important de noter que la couche 1 est divisée en 2 ensembles: le premier, à la base, est constitué de blocs accumulés sur le substratum, et qui sont toujours grossiers quelque soit le contexte; le second, au sommet, de texture plus fine et contenant les concrétions calcaires, traduisant un épisode de solifluxion en milieu froid, (Kervazo, communication personnelle).

(2) Cet ensemble comprend également les niveaux de base de la séquence (couches 1 à 3 ).

(3) Ce qui confirme par ailleurs que les "épisodes" de Laugerie et de Lascaux correspondent à de simples variations d'humidité dans un contexte steppique, (Bosselin et Djindjian, 1997a et b).

(4) H. Laville place l'oscillation de Bølling entre les couches 20 et 21 , Arl. Leroi-Gourhan au milieu de la couche 20, les couches 21 à 23 étant alors attribuées au Dryas II, (Laville, 1986; Arl. Leroi-Gourhan, 1986). Ch. Leroyer (communication personnelle) reconnaît dans la phase à Juniperus des couches 21 sup. à 23 le témoin caractéristique du premier repeuplement de la forêt sur la steppe tardiglaciaire, et correspondant à l'oscillation de Bølling.

(5) Il convient de noter que le niveau de base (couche 1), attribué par la suite au Proto-Solutréen ( $c f$. infra), appartient également à cet ensemble.

(6) L'attribution des industries aurignacoïdes inter Gravetto-Solutréennes (ex-Aurignacien final) à un faciès de transition entre Gravettien final et Solutréen a fait l'objet de nombreuses études et publications depuis le congrès S.P.F. de Carcassonne en 1994. À cette occasion, deux communications (Djindjian, sous presse et Zilhao, Aubry et Almeida, sous presse) ont proposé simultanément et indépendamment cette attribution : le premier auteur, à partir d'un réexamen des données Laugerie-Haute (Aurignacien V), de la Salpétrière (Aurignacien moyen/terminal) et de niveaux aurignacoïdes de sites cantabriques (la Riera, el Pendo), de Catalogne (Reclau-Viver) et du Levant (Mallaetes, Barranc-Blanc); les seconds auteurs à partir d'un réexamen du site de Laugerie-Haute et de données nouvelles de l'abri Casserole aux Eyzies et de sites portugais (Cabeço de Porto Marinho II, III ; Gato Preto ; Lapa do Anecrial).

Le congrès U.I.S.P.P. de Forli en 1996 a fait l'objet d'un colloque consacré à l'Aurignacien récent ("Late Aurignacian") dont les orga- nisateurs ont invité F. Bazile, F. Djindjian et A. Marks notamment, pour présenter une synthèse de la question sur respectivement le Languedoc, l'Aquitaine et le Portugal, (Bazile, 1996 ; Djindjian, 1996 ; Marks et Almeida, 1996).

Consécutivement à ces communications aux congrès de Carcassonne et de Forli, plusieurs articles ont été plus ont moins rapidement publiés, (Aubry et al., 1995; Bosselin et Djindjian, 1997a et b ; Zilhao et Aubry, 1995).

(7) Ce deuxième axe factoriel révèle en fait une erreur de codification due à la typologie. En effet, les variables sont présentées sous la forme de modalités n'étant pas rigoureusement exclusives entre elles sagaie à base en biseau simple - sagaie de section ovalaire, sans prendre en compte l'existence de sagaies à biseau simple, de section ovalaire. En conséquence, cet axe ne sera pas pris en compte dans la classification automatique.

(8) Entre environ 21000 B.P. et 20000 B.P., entre environ 19000 B.P. et 18000 B.P., entre environ $15500-16000$ B.P. et 13500 B.P., aux environs de 12000 B.P. (? : si on admet encore l'existence du Dryas II) et entre environ 10800 B.P. et 10000 B.P.

(9) Ce ravinement entre le Solutréen et le Badegoulien se retrouve également (Hoyos-Gomez, 1995) à la Viña (inter IV/V), la Lluera (érosion de VI), Cova Rosa (inter DE/D) et Las Caldas (inter 6/7), et peut avoir provoqué ponctuellement des mélanges d'industries et/ou de sédiments.

(10) Pour le Solutréen : feuilles de laurier et pointes à cran solutréennes en Aquitaine, feuilles de laurier asymétriques et pointes à base concave dans les Pyrénées et en Cantabres, "Épigravettien" ancien à foliacés puis à crans en Italie et en Provence (Arénien), pointes à cran méditerranéennes et lamelles à dos en Languedoc et en Catalogne, pointes à pédoncule et ailerons et pointes à cran méditerranéennes sur le levant espagnol. Pour le Badegoulien : burins transversaux puis raclettes en Périgord, burins transversaux puis raclettes et enfin raclettes et lamelles à dos dans le Quercy (et en Languedoc occidental ?), foisonnement des raclettes en périphérie de l'Aquitaine (Gironde, Indre), encoches et denticulés dans les Cantabres, grattoirs aurignaciens, lames retouchées et "outils archaïques" sur le levant espagnol.

\section{RÉFÉRENCES BIBLIOGRAPHIQUES}

ADAN-ALVAREZ G.E. (1994) - El material óseo del Solutrense peninsular : secuencia, soporte óseo, métodos de trabajo y tipologia. Férvedes, 1, p. 159-174.

ALTUNA J. (1986) - The mammalian faunas from the prehistoric site of La Riera, in : La Riera Cave. Stone Age Hunter-Gatherer adaptations in Northern Spain, L.G. Straus et G.A. Clark ed., Anthropological Research Papers, 36, p. 237-274.

ALTUNA J. et MERINO J.M. (1984) - El yacimiento prehistorico de la Cueva de Ekain (Deba, Guipúzcoa), Sociedad de Estudios Vasco, Serie B1, San Sebastian.

ALTUNA J. et alii (1985) - Cazadores magdaleniense en la Cueva de Erralla (Cestona, Pais Vasco), Munibe, 37, San Sebastian.

ALTUNA J. et alii (1990) - La Cueva de Amalda (Zestoa, País Vasco). Ocupaciones Paleolíticas y Postpaleolíticas, Sociedad de Estudios Vasco, Serie B4, San Sebastian.

ARESO P. (1984) - El yacimiento de la Cueva de Ekain (Deba, Guipúzcoa). Sedimentología de los niveles VII a II, in: El yacimiento prehistorico de la Cueva de Ekain (Deba, Guipúzcoa), J. Altuna et J.M. Merino, Sociedad de Estudios Vasco, Serie B1, p. 47-60.

AUBRY T. et alii (1995) - Les niveaux intermédiaires entre le Gravettien et le Solutréen de l'abri Casserole (Les Eyzies de Tayac) : mise en évidence d'un mode de production original des microlithes-Implications, Bulletin de la Société Préhistorique Française, t. 92 (3), p. $296-301$.

AURA-TORTOSA J.E. (1995) - El Magdaleniense Mediterráneo : La Cova del Parpalló (Gandia, Valencia), Servicio de Investigación Prehistórica, Serie de Trabajos Varios, 91, Valencia.

BARANDIARAN-MAETZU I. (1996) - Le Paléolithique Supérieur au Pays Basque et dans le Bassin de l'Ebre, in: Le Paléolithique
Supérieur Européen. Bilan Quinquennal 1991-1996, M. Otte, ERAUL 76, p. 319-322.

BARANDIARAN-MAETZU I. et alii (1985) - Excavaciones en la Cueva del Juyo, Centro de Investigaciones y Museo de Altamira, 14, Madrid.

BAZILE F. (1996) - La question de "1'Aurignacien terminal" en Languedoc, in: $13^{e}$ congrès U.I.S.P.P., Forli, 1996, vol. 6: "The Upper Palaeolithic ", A. Palma di Cesnola, A. Montet-White et K. Valoch éd., éd. ABACO, p. 55-68.

BERNALDO DE QUIROS F. (1982) - Los inicios del Paleolitico supe rior cantábrico, Centro de Investigaciones y Museo de Altamira, 8, Madrid.

BERNALDO DE QUIROS F. (1996) - Le Paléolithique Supérieur dans la région Cantabrique (1991-1995), in: Le Paléolithique Supérieur Européen. Bilan Quinquennal 1991-1996, M. Otte, ERAUL 76, p. 323-327.

BICHO N.F. (1997) - Spatial, technological, and economic organisation after the Last Glacial Maximum in Portuguese Prehistory, in: El món mediterrani després del pleniglacial (18000-12000 $B P)$, J. Fullola i N. Soler, Museu d'arqueologia de Catalunya, Centre d'Investigacions Arqueològiques, Monografià 17 , Girona, pp. 213-223.

BORDES F. (1958) - Nouvelles fouilles à Laugerie-Haute Est. Premiers résultats, L'Anthropologie, 62 (5-6), p. 205-244.

BOSSELIN B. et DJINDJIAN F. (1988) - Un essai de structuration du Magdalénien français à partir de l'outillage lithique, Bulletin de la Société Préhistorique Française, t. 85 (10-12), p. 304-331.

BOSSELIN B. et DJINDJIAN F. (1997a) - L'Aurignacien tardif : un faciès de transition du Gravettien au Solutréen! Préhistoire Européenne, 10, p. 107-125. 
BOSSELIN B et DJINDJIAN F. (1997b) - Une révision du Solutréen de Laugerie-Haute et le problème des transitions GravettienSolutréen et Solutréen-Badegoulien en Aquitaine, Bulletin de la Société Préhistorique Française, t. 94 (4), p. 443-454.

BOYER-KLEIN A. (1980) - Nouveaux résultats palynologiques de sites solutréens et magdaléniens cantabriques, Bulletin de la Société Préhistorique Française, t. 77, p. 103-107.

BOYER-KLEIN A. (1981) - Análisis palinológico del Rascaño, in : El Paleolitico Superior de la Cueva del Rascaño (Santander), J. Gonzales-Echegaray et I. Barandiaran-Maetzu, Centro de Investigaciones y Museo de Altamira, 3, p. 217-220.

BOYER-KLEIN A. (1985) - Analyse pollinique de la grotte d'Erralla, in: Cazadores magdaleniense en la Cueva de Erralla (Cestona, Pais Vasco), J. Altuna et alii, Munibe, 37, p. 45-48.

BOYER-KLEIN A. et LEROI-GOURHAN Arl. (1985) - Análisis palinológico de la Cueva de Juyo, in: Excavaciones en la Cueva del Juyo I, Barandiaran-Maetzu et alii, Centro de Investigaciones y Museo de Altamira, 14, p. 55-61.

BUTZER K.W. (1981) - Cave Sediments, Upper Pleistocene stratigraphy and Mousterian facies in Cantabrian Spain, Journal of Archeological Science, 8, p. 133-183.

CABRERA-VALDES V. (1977) - El yacimiento solutrense de Cueva Chufin (Riclones, Santander), Actas del XIV Congreso Nacional de Arqueologia, p. 157-164

CABRERA-VALDES V. (1984) - El yacimiento de la Cueva del Castillo (Puente Viesgo, Santander), Bibliotheca Praehistorica Hispaña, 22, Madrid.

CORCHON-RODRIGUEZ Ma.S. (1981) - Cueva de Las Caldas. San Juan de Priorio (Oviedo), Excavaciones Arqueologicas en Espan̄a, 115, Madrid.

CORCHON-RODRIGUEZ Ma.S. (1990) - La Cueva de Las Caldas (Priorio, Oviedo). Investigaciones efectuadas entre 1980 y 1986 , Excavaciones Arqueologicas en Asturias, 1, p. 37-54.

CORCHON-RODRIGUEZ Ma.S. (1992) - La Cueva de Las Caldas (Priorio, Oviedo). Investigaciones efectuadas entre 1987 y 1990, Excavaciones Arqueologicas en Asturias, 2, p. 33-47.

CORCHON-RODRIGUEZ Ma.S. (1993-94) - El Magdaleniense con triángulos de Las Caldas (Asturias, España), Nuevos datos para la definicíon del Magdaleniense inferior Cantábrico. Zephyrus, XLVI, p. 77-94.

CORCHON-RODRIGUEZ Ma.S. (1994) - Arte Mobiliar e industria ósea solutrense en la cornisa cantábrica, Férvedes, 1, p. 131-148.

CORCHON-RODRIGUEZ Ma.S. (1995) - La Cueva de Las Caldas (Priorio, Oviedo). Investigaciones efectuadas entre 1991 y 1994, Excavaciones Arqueologicas en Asturias, 3, p. 45-60.

DJINDJIAN F. (1996) - Les industries aurignacoïdes en Aquitaine entre 25000 et $15000 \mathrm{BP}$, in: $13^{\circ}$ congrès U.I.S.P.P. Forli, 1996, vol. 6: "The Upper Palaeolithic", A. Palma di Cesnola, A. Montet-White et K. Valoch éd., éd. ABACO, p. 41-54.

DUPRE M. (1984) - Palinología de los niveles VII a II del yacimiento de Ekain, in: El yacimiento prehistorico de la Cueva de Ekain (Deba, Guipúzcoa), J. Altuna et J.M. Merino, Sociedad de Estudios Vasco, Serie B1, p. 61-63.

FORTEA-PEREZ J. (1990) - Abrigo de la Viña. Informe de las campañas 1980-1986, Excavaciones Arqueologicas en Asturias, 1, p. $55-68$.

FORTEA-PEREZ J. (1992) - Abrigo de la Viña. Informe de las campañas 1987 a 1990, Excavaciones Arqueologicas en Asturias, 2, p. $19-28$.

FORTEA-PEREZ J. (1995) - Abrigo de la Viña. Informe de las campañas 1991 a 1993, Excavaciones Arqueologicas en Asturias, 3, p. 19-32.

FORTEA-PEREZ J. (1996) - Le Paléolithique Supérieur en Espagne : Galice et Asturies (1991-1995), in: Le Paléolithique Supérieur
Européen. Bilan Quinquennal 1991-1996, M. Otte, ERAUL To, p. $329-344$.

FORTEA-PEREZ J. et alii (1992) - La Cueva de Llonin (Llonin, Peñamellera Alta). Campañas 1987 a 1990, Excavaciones Arqueologicas en Asturias, 2, p. 9-18.

FORTEA-PEREZ J. et alii (1995) - La Cueva de Llonín (Llonín, Peñamellera Alta). Campañas 1991 a 1994, Excavaciones Arqueologicas en Asturias, 3, p. 33-43.

GONZALES-MIRALES M.R. (1986) - La Riera : Bone and Antler artifact assemblages, in: La Riera Cave. Stone Age HunterGatherer adaptations in Northern Spain, L.G. Straus et G.A. Clark, Anthropological Research Papers, 36, p. 209-218.

GONZALES-ECHEGARAY J. et BARANDIARAN-MAETZU I. (1981) - El Paleolitico superior de la Cueva del Rascaño (Santander), Centro de Investigaciones y Museo de Altamira, 3, Santander.

GONZALES-ECHEGARAY I. et FREEMAN L.G. (1971-1973) Cueva Morin: Excavaciones 1966-1968/1969, Patronato de las Cuevas Prehistóricas, Santander.

HOYOS-GOMEZ M. (1981) - Estudio geológico y sedimentológico de la Cueva de Las Caldas, in: Cueva de Las Caldas. San Juan de Priorio (Oviedo), Ma.S. Corchon-Rodriguez, Excavaciones Arqueologicas en España, 115, p. 11-55.

HOYOS-GOMEZ M. (1995) - Paleoclimatología del Tardiglacial en la cornisa Cantábrica basada en los resultados sedimentológicos de yacimientos arqueológicos kársticos, in : El final del Paleolítico Cantábrico, A. Moure-Romanillo et alii, Universita de Cantábria, p. 15-75.

HOYOS-GOMEZ M. et FUMANAL M.P. (1985) - La Cueva de Erralla : Estudios sedimentológico, in: Cazadores magdaleniense en la Cueva de Erralla (Cestona, Pais Vasco), J. Altuna et alii, Munibe, 37, p. 29-43.

HOYOS-GOMEZ M. et RASILLA-VIVES M. de la (1994) - Dataciones $\mathrm{C}^{14}$ del Paleolítico superior del abrigo de Cueto de la Mina (Posada de Llanes, Asturias), Trabajos de Prehistoria, 51 (2), p. 143-147

HOYOS-GOMEZ M. et alii (1980) - La Cueva de la Paloma, Soto de Regueras (Asturias), Excavaciones Arqueologicas en España, 116, Madrid.

LAVILLE H. (1986) - Stratigraphy, Sedimentology and Chronology of the La Riera Cave Deposits, in: La Riera Cave. Stone Age Hunter-Gatherer adaptations in Northern Spain, L.G. Straus et G.A. Clark, Anthropological Research Papers, 36, p. 25-55.

LAVILLE H. et HOYOS-GOMEZ M. (1981) - Estudios geológicos de la Cueva de Rascaño, in : El Paleolitico Superior de la Cueva del Rascaño (Santander), J. Gonzales-Echegaray et I. BarandiaranMaetzu: " ", Centro de Investigaciones y Museo de Altamira, 3, p. $189-210$.

LEROI-GOURHAN Arl. (1986) - The Palynology of La Riera Cave, in: La Riera Cave. Stone Age Hunter-Gatherer adaptations in Northern Spain, L.G. Straus et G.A. Clark, Anthropological Research Papers, 36, p. 59-64.

LEROI-GOURHAN Arl. (1971) - Análisis polinico de Cueva Morin, in: Cueva Morin: Excavaciones 1966-1968, J. Gonzales-Echegaray et L.G. Freeman, Patronato de las Cuevas Prehistóricas, p. $359-365$

LOPEZ P. (1982) - Abauntz: Análisis polínico, Trabajos de Arqueología Navarra, 3, p. 355-358.

MARKS A.E. et ALMEIDA F. (1996) - The Late Aurignacian and "Aurignacian" elements in the Upper Palaeolithic of the Portuguese Estramadoura, Portugal, in: $13^{c}$ congrès U.I.S.P.P., Forli, 1996, vol. 6: "The Upper Palaeolithic", A. Palma di Cesnola, A. Montet-White et K. Valoch éd., éd. ABACO, p. 11-22.

MARKS A.E. et MISHOE M.B. (1997) - The Magdalenian of Portuguese Estremandura, in: El món mediterrani després del pleniglacial (18000-12000 BP), J. Fullola i N. Soler, Museu 
d'arqueologia de Catalunya, Centre d'Investigacions Arqueològiques, Monografià 17, Girona, p. 225-232.

MOURE-ROMANILLO A. et alii (éd.) (1995) - El Final del Paleolitico cantábrico, Universidad de Cantábria.

RASILLA-VIVES M. de la (1989) - Secuncia y crono-estratigrafia del Solutrense cantábrico, Trabajos de Prehistoria, 46, p. 35-46.

RASILLA-VIVES M. de la (1990a) - Cueto de la Mina. Campañas 1981-1986, Excavaciones Arqueologicas en Asturias, 1, p. 79-86.

RASILLA-VIVES M. de la (1990b) - Le Solutréen cantabrique, in : Feuilles de Pierre, M. Otte, ERAUL 42, p. 481-484.

RASILLA-VIVES M. de la (1994a) - La cronología radiométrica del Solutrense en la Península Ibérica y sus correlación crono-climática, Férvedes, 1, p. 57-67.

RASILLA-VIVES M. de la (1994b) - El Solutrense de la cornisa cantábrica, Férvedes, 1, p. 69-87.

RASILLA-VIVES M. (de la) éd. (1994) - Monográfico el Solutrense en la península ibérica, Férvedes, 1, Museo de Prehistoxia e Arqueoloxia de Villalba.

RODRIGUEZ-ASENSIO. J.A. (1990) - Excavaciones arqueológicas realizad en la Cueva de "La Lluera" (San Juan de Priorio, Oviedo), Excavaciones Arqueologicas en Asturias, 1, p. 15-17.

RODRIGUEZ-ASENSIO. J.A. (1992) - Excavaciones arqueológicas en la Cueva de La Lluera II. San Juan de Priorio. Oviedo, Excavaciones Arqueologicas en Asturias, 2, p. 29-32.

STRAUS L.G. (1975) - $i$ Solutrense o Magdaleniense inferior cantábrico? Significado de las diferencias, Boletiin del Instituto de Estudios Asturianos, 86, p. 781-790.

STRAUS L.G. (1983) - El Solutrense vasco-cantábrico: una nueva perspectiva, Centro de Investigaciones y Museo de Altamira, 10 Madrid.

STRAUS L.G. (1986a) - An overview of the La Riera chronology, in : La Riera Cave. Stone Age Hunter-Gatherer adaptations in Northern Spain, L.G. Straus et G.A. Clark, Anthropological Papers Research, 36, p. 19-23.

STRAUS L.G. (1986b) - La Riera and the terminal pleistocene environments of Cantábrian Spain, in: La Riera Cave. Stone Age Hunter-Gatherer adaptations in Northern Spain, L.G. Straus et G.A. Clark, Anthropological Papers Research, 36, p. 67-73.

STRAUS L.G. (1986c) - A comparison of La Riera assemblages with those from contemporary sites in Cantábrian Spain, in: La Riera Cave. Stone Age Hunter-Gatherer adaptations in Northern Spain, L.G. Straus et G.A. Clark, Anthropological Papers Research, 36 p. 219-236.

STRAUS L.G. et alii (1986) - Patterns of lithic raw material variations at La Riera, in : La Riera Cave. Stone Age Hunter-Gatherer adaptations in Northern Spain, L.G. Straus et G.A. Clark, Anthropological Papers Research, 36, p. 189-208.

STRAUS L.G. et CLARK G.A. éd. (1986) - La Riera Cave. Stone Age Hunter-Gatherer adaptations in Northern Spain, Anthropological Papers Research, 36, Arizona State University, Tempe.
STRAUS L.G. et CLARK G.A. (1986a) - Introduction : research design, site location and setting, History of earlier excavations and Methodology, in : La Riera Cave. Stone Age Hunter-Gatherer adaptations in Northern Spain, L.G. Straus et G.A. Clark, Anthropological Papers Research, 36, p. 1-17.

STRAUS L.G. et CLARK G.A. (1986b) - La Riera : Archaeological remains - Level content and characterisation, in : La Riera Cave. Stone Age Hunter-Gatherer adaptations in Northern Spain, L.G. Straus et G.A. Clark, Anthropological Papers Research, 36, p. $75-187$.

STRAUS L.G. et CLARK G.A. (1986c) - Synthesis and conclusions Part II : The La Riera excavation, chronostratigraphy, paleoenvironments and cultural sequence in perspective in: La Riera Cave. Stone Age Hunter-Gatherer adaptations in Northern Spain, L.G. Straus et G.A. Clark, Anthropological Papers Research, 36, p. 367-383.

TROTIGNON F. (1984) - Études sur l'abri Fritsch (Indre), XIX ${ }^{\circ}$ suppl. à Gallia Préhistoire, Paris.

UTRILLA-MIRANDA P. (1981) - El Magdaleniense inferior y Medio en la Costa Cantabrica, Centro de Investigaciones y Museo de Altamira, 4, Santander.

UTRILLA-MIRANDA P. (1982) - El yacimiento de la Cueva de Abauntz (Arraiz, Navarra), Trabajos de Arqueología Navarra, 3 , p. 203-355.

UTRILLA-MIRANDA P. (1986) - La varilla pseudoexcisa de Aitzbitarte IV y sus paralelos Franceses, in Estudios en Homenaje a Antonio Beltrán, Zaragoza, p. 205-225.

UTRILLA-MIRANDA P. (1989) - El Magdaleniense inferior en la costa Cantábrica, in: Le Magdalénien en Europe, M. Otte, ERAUL, 38, p. $399-418$

VEGA DEL SELLA el Conde de la (1930) - Las Cuevas de la Rieray Balmori (Asturias), Comisión de Investigaciones Paleontológicas y Prehistóricas, 38, Madrid.

ZILHAO J. (1997) - The Paleolithixc settlement of Portuguese Estramadure after the last glacial maximum, in: El món mediterrani després del pleniglacial (18000-12000 BP), J. Fullola i N. Soler, Museu d'arqueologia de Catalunya, Centre d'Investigacions Arqueològiques, Monografià 17, Girona, p. 233-242.

ZILHAO J. et AUBRY T. (1995) - The point of Vale Comprido and the origins of the Solutrean, L'Anthropologie, 99 (1), p. 125-142.

Bruno BOSSELIN

4, place des Vernes - F-77500 Chelles

François DJINDJIAN

U.P.R. 315 du C.N.R.S.

27, rue Damesme - F-75013 Paris 